\title{
Quantum process tomography of excitonic dimers from two-dimensional electronic spectroscopy. I. General theory and application to homodimers
}

\section{Citation}

Yuen-Zhou, Joel, and Ala\#n Aspuru-Guzik. 2011. “Quantum Process Tomography of Excitonic Dimers from Two-Dimensional Electronic Spectroscopy. I. General Theory and Application to Homodimers." The Journal of Chemical Physics 134 (13): 134505.

\section{Published Version}

doi:10.1063/1.3569694

\section{Permanent link}

http://nrs.harvard.edu/urn-3:HUL.InstRepos:12410516

\section{Terms of Use}

This article was downloaded from Harvard University's DASH repository, and is made available under the terms and conditions applicable to Other Posted Material, as set forth at http:// nrs.harvard.edu/urn-3:HUL.InstRepos:dash.current.terms-of-use\#LAA

\section{Share Your Story}

The Harvard community has made this article openly available.

Please share how this access benefits you. Submit a story.

\section{Accessibility}




\title{
Quantum process tomography of excitonic dimers from two-dimensional electronic spectroscopy. I. General theory and application to homodimers
}

\author{
Joel Yuen-Zhou and Alán Aspuru-Guzik ${ }^{\text {a) }}$ \\ Department of Chemistry and Chemical Biology, Harvard University, Cambridge, Massachusetts 02138, USA
}

(Received 14 January 2011; accepted 3 March 2011; published online 7 April 2011)

\begin{abstract}
Is it possible to infer the time evolving quantum state of a multichromophoric system from a sequence of two-dimensional electronic spectra (2D-ES) as a function of waiting time? Here we provide a positive answer for a tractable model system: a coupled dimer. After exhaustively enumerating the Liouville pathways associated to each peak in the 2D-ES, we argue that by judiciously combining the information from a series of experiments varying the polarization and frequency components of the pulses, detailed information at the amplitude level about the input and output quantum states at the waiting time can be obtained. This possibility yields a quantum process tomography (QPT) of the single-exciton manifold, which completely characterizes the open quantum system dynamics through the reconstruction of the process matrix. In this manuscript, we present the general theory as well as specific and numerical results for a homodimer, for which we prove that signals stemming from coherence to population transfer and vice versa vanish upon isotropic averaging, therefore, only allowing for a partial QPT in such case. However, this fact simplifies the spectra, and it follows that only two polarization controlled experiments (and no pulse-shaping requirements) suffice to yield the elements of the process matrix, which survive under isotropic averaging. Redundancies in the 2D-ES amplitudes allow for the angle between the two site transition dipole moments to be self-consistently obtained, hence simultaneously yielding structural and dynamical information of the dimer. Model calculations are presented, as well as an error analysis in terms of the angle between the dipoles and peak amplitude extraction. In the second article accompanying this study, we numerically exemplify the theory for heterodimers and carry out a detailed error analysis for such case. This investigation reveals an exciting quantum information processing (QIP) approach to spectroscopic experiments of excitonic systems, and hence, bridges an important gap between theoretical studies on excitation energy transfer from the QIP standpoint and experimental methods to study such systems in the chemical physics community. (C) 2011 American Institute of Physics. [doi:10.1063/1.3569694]
\end{abstract}

\section{INTRODUCTION}

Multidimensional optical spectroscopies (MDOS) provide very powerful tools to study excited state dynamics of multichromophoric systems in condensed phases. These techniques distribute spectral features along several dimensions, uncluttering data which would otherwise appear obscured in linear spectroscopies and simultaneously yielding novel information on the dynamics of the probed system. ${ }^{1}$ Possibilities in multidimensional techniques include decongesting spectral line shapes, differentiating between homogeneous and inhomogeneous broadening mechanisms, providing unambiguous signatures about couplings between chromophores, and yielding signatures of coherent and incoherent processes involving excited states at the amplitude level. ${ }^{2,3}$ Although MDOS have been historically inspired by their NMR analogues, the time scales of the physical and chemical processes studied through MDOS are quite different from the ones in NMR. ${ }^{4-7}$ The characteristic time scales of NMR are milliseconds, a resolution that does not allow for the observation of a wide variety of chemical dynamics in condensed phases occurring in the orders of femto and picoseconds. On the other hand, femtosecond time scales can be easily accessed with ultrafast op-

${ }^{a)}$ Electronic mail: aspuru@chemistry.harvard.edu. tical techniques. Examples of phenomena studied via MDOS are vast and include molecular reorientation processes, ${ }^{8,9}$ electron transfer, ${ }^{10}$ vibrational coherences in organometallic complexes ${ }^{11-13}$ or halogens in rare gas matrices, ${ }^{14,15}$ phonon dynamics in carbon nanotubes, ${ }^{16}$ protein unfolding kinetics ${ }^{17}$ and organic polymers, ${ }^{18,19}$ many-body physics in semiconductor quantum wells ${ }^{20-22}$ and quantum dots, ${ }^{23}$ as well as excitonic dynamics in light-harvesting systems. ${ }^{24-29}$ In particular, experiments on the latter topic have revealed the presence of unexpectedly long lived quantum coherences in photosynthetic systems. This phenomenon has attracted considerable attention from the quantum information processing (QIP) community, which has focused on elucidating the interplay between coherent and incoherent dynamics in the transport of energy in excitonic networks. ${ }^{30-37}$

Traditionally, the spectroscopy of condensed phases is formulated as a response problem: The molecular system is perturbed with a sequence of short laser pulses, and the coherent polarization response due to this set of perturbations (nonlinear polarization) is subsequently measured. ${ }^{1}$ Informally, we can describe the exercise as "kicking" the quantum black box (molecular system) and "listening to the whispers" (measuring the response) due to the kicks, from which some properties of the box can be inferred. This description of 
spectroscopy is reminiscent to an idea stemming from the quantum optics and QIP communities, namely, quantum process tomography (QPT). ${ }^{38-41}$ Broadly speaking, QPT is a systematic procedure to characterize a quantum black box by sending a set of inputs, measuring their outputs, and analyzing the functional relationships between them. With the increasing effort of quantum engineering of gates and devices, QPT constitutes a cornerstone of QIP theory and experiment, as it provides a necessary check on the performance of the respective quantum black boxes. A natural question arises from the comparison of the two aforementioned concepts: Can the spectroscopy of condensed phases be formulated as a QPT? In a previous study, ${ }^{42}$ we provided an affirmative answer to this question, at least for a molecular dimer. We showed that a set of two-color polarization controlled rephasing photon-echo (PE) experiments are sufficient to reconstruct the density matrix elements associated with the dynamics of the single-exciton manifold, and therefore, systematically characterize the excited state dynamics of the dimer, which can be regarded as the black box. For pedagogical reasons, we found it simpler and more convenient to concentrate our attention on the real time picture of the experiment, to make an explicit identification of the preparation, evolution, and detection steps of the QPT, with the coherence, waiting, and echo times, respectively. However, due to the widespread practice of displaying partially Fourier transformed data of the nonlinear optical polarization with respect to certain time intervals, it is worthwhile translating our results to the more visual two-dimensional electronic spectrum ${ }^{5,115}$ (2D-ES), and in fact, this is one of the main results of the present work.

The present article is organized as follows: we begin in Sec. II with a review of some relevant ideas of QPT and also introduce the process matrix as the main object to be reconstructed by means of QPT. In Sec. III, relevant details on the dimer model system are presented. Section IV describes the rephasing heterodyne photon-echo experiment for the dimer and explains that the collected macroscopic polarization signal is a linear combination of elements of the process matrix at the waiting time $\chi(T)$. This implies that QPT can be performed by repeating several experiments with different pulse parameters in order to extract these elements. In Sec. V, the ideas of Sec. IV are mapped into the language of a 2D-ES, where each of the diagonal and cross peaks is associated with a set of elements of $\chi(T)$, and each of the axes of the spectrum can be associated with a preparation and a detection stage. Finally, in Sec. VI, these ideas are specialized to homodimer systems where, after isotropic averaging, only a partial QPT is possible, as some elements of $\chi(T)$ are undetectable. Nevertheless, we note that the partial QPT is easily realized with current experimental capabilities, since it can be reconstructed with only two spectra resulting from different pulse polarization configurations for each given waiting time. The angle between the site dipoles is self-consistently obtained from these spectra, and an error analysis based on this angle as well as peak overlaps is carried out. Numerical calculations on a secular Redfield dissipation model are presented. Extensions of the procedure to account for inhomogeneous broadening, more sophisticated signal analysis, as well as bigger systems, are discussed at the end of this manuscript.
A detailed analysis for heterodimers is carried out in the next article accompanying this investigation. The present work is an important bridge between the QIP approach to study of energy transport in excitonic networks and the actual experimental techniques utilized to probe such systems in the chemical physics community.

\section{RELEVANT CONCEPTS OF QUANTUM PROCESS TOMOGRAPHY AND GENERAL DEFINITIONS}

Consider an arbitrary quantum system (quantum black box) interacting with an environment. We are interested in its evolution as a function of time $T$ in the form of a reduced density matrix $\rho(T)$. Very generally, this evolution is a linear transformation acting on the initial quantum state (see Appendix): ${ }^{43,44}$

$$
\rho(T)=\chi(T) \rho(0) .
$$

$\chi(T)$ is the central object of this article and shall be called process matrix. Equation (1) can be regarded as an integrated equation of motion for every $T .{ }^{117}$ It is important to emphasize that the expression holds both for Markovian and nonMarkovian dynamics, as can be seen from the derivation in the Appendix. Equation (1) can be expressed in terms of a basis for the Liouville space of the system:

$$
\rho_{a b}(T)=\sum_{c d} \chi_{a b c d}(T) \rho_{c d}(0)
$$

For purposes of this article, we present two useful definitions. Consider the Liouville space $\mathcal{L}$ of the system and classify the vectors of $\mathcal{L}$ in proper and improper density matrices. A state or a density matrix is proper if it satisfies all the conditions of a physical quantum state; namely, this is Hermitian, positive semidefinite, and has trace one. An improper state is any matrix that lives in the Liouville space but is not a proper density matrix. Clearly, any improper density matrix in the same Liouville space may be written as a unique linear combination of a set of linearly independent proper density matrices. In principle, Eq. (2), being a physical equation of motion, is restricted to the domain of proper density matrices $\rho(0)$. However, by linearity, its extension to any linear combination of proper states is well defined, so its validity for improper density matrices is not under question.

The meaning of the process matrix $\chi(T)$ is easy to grasp: Conditional on the initial state being prepared at $\rho(0)=|c\rangle\langle d|, \chi_{a b c d}(T)$ is the value of the entry $a b$ of the quantum state after time $T, \rho(T)$, i.e., $\chi_{a b c d}(T)=\langle a|\rho(T)| b\rangle$. Therefore, $\chi_{a b c d}(T)$ denotes a state to state transfer amplitude. Note that $\rho(0)=|c\rangle\langle d|$ is an improper density matrix if $c \neq d$ (coherences on their own are not valid quantum states). However, improper states are not necessarily unphysical as one expects at a first glance. Most of our intuition for nonlinear spectroscopies in the perturbative regime stems from the consideration of how a perturbative amplitude created at a certain entry $|c\rangle\langle d|$ of the total (proper) density matrix is transferred to other entries due to free evolution, as time progresses. ${ }^{1}$ It is not the evolution of the total density matrix (which to leading order is unperturbed, mostly in 
its ground state, and not yielding a time-dependent dipole) what is effectively monitored in the phase-matched signal, but the evolution of an effective density matrix, such as $|c\rangle\langle d|$, which can be improper. Terms such as transfer from population to population, coherence to coherence, population to coherence, and coherence to population are all ubiquitous in the jargon of MDOS. However, the monitoring of these terms is often ambiguous, incomplete, and in most cases, qualitative. Obtaining quantitative information about these events amounts to finding each of the elements of $\chi(T)$.

The transformation in Eq. (2) is limited by two classes of restrictions for the process matrix associated with Hermiticity and trace preservation:

$$
\begin{gathered}
\sum_{a} \chi_{a a c d}(T)=\delta_{c d}, \\
\chi_{a b c d}(T)=\chi_{b a d c}^{*}(T) .
\end{gathered}
$$

We derive these conditions in the Appendix, but their content is intuitive: if $\rho(0)$ is a proper density matrix, $\rho(t)$ remains as a valid quantum state as $T$ evolves if these two requirements are preserved. In particular, elements of the form $\chi_{a a b b}(T)$, which denote population transfers, are purely real as one expects, whereas the other elements are in general complex. ${ }^{118}$

Equations (1) and (2) are remarkable because they guarantee that, in principle, if $\chi(T)$ is known, the quantum black box described by $\rho(T)$ is perfectly understood, as it predicts by linearity the evolution of an arbitrary initial state in $\mathcal{L}$. Although $\rho(T)$ describes an open quantum system, details about the environment evolution need not be included explicitly, but only in an averaged sense via the elements of $\chi(T)$. We shall operationally define $Q P T$ as any procedure to reconstruct $\chi(T)$. A possible QPT is the following: (a) Prepare a linearly independent set of states $\rho(0)$ that spans $\mathcal{L}$; (b) for each of the prepared states, wait for a free evolution time $T$ and determine the density matrix at that time. Any protocol for determining a density matrix for a system is called quantum state tomography (QST). ${ }^{45-49}$ In essence, QPT can be carried out for any system if both a selective preparation of initial states and QST can be achieved. Variants of this methodology exist although all of them operate within the same spirit. ${ }^{38-41}$ QPT has been successfully implemented in a wide variety of experimental scenarios, including nuclear magnetic resonance, ${ }^{50-52}$ ion traps, ${ }^{53}$ single photons, ${ }^{54,55}$ solid state qubits, ${ }^{56}$ optical lattices, ${ }^{57}$ and Josephson junctions. ${ }^{58}$ In this article, we show how to perform QPT for a model coupled dimer using twocolor polarization controlled heterodyne photon-echo experiments, extending the domain of application of QPT to systems of chemical and biophysical interest.

\section{MODEL SYSTEM: COUPLED DIMER}

Consider a molecular dimer described by the effective Hamiltonian: $3,59,60$

$$
H_{S}=\omega_{A} a_{A}^{+} a_{A}+\omega_{B} a_{B}^{+} a_{B}+J\left(a_{A}^{+} a_{B}+a_{B}^{+} a_{A}\right),
$$

where $a_{i}^{+}$and $a_{i}$ are creation and annihilation operators for a single Frenkel exciton in the site $i \in\{A, B\}, \omega_{A}, \omega_{B}$ are the first and second site energies, and $J$ is the coupling between the chromophores.

The standard diagonalization of this Hamiltonian, which is effectively a two-level system for the single-exciton manifold, follows from defining some convenient parameters: The average of the site energies $\bar{\omega}=1 / 2\left(\omega_{A}+\omega_{B}\right)$, the difference $\Delta=1 / 2\left(\omega_{A}-\omega_{B}\right)$, and the mixing angle $\theta=1 / 2 \arctan \left(\frac{J}{\Delta}\right)$. By introducing the operators

$$
\begin{aligned}
& a_{\alpha}=\cos \theta a_{A}+\sin \theta a_{B}, \\
& a_{\beta}=-\sin \theta a_{A}+\cos \theta a_{B},
\end{aligned}
$$

the Hamiltonian in Eq. (5) can be readily written as

$$
H_{S}=\omega_{\alpha} a_{\alpha}^{+} a_{\alpha}+\omega_{\beta} a_{\beta}^{+} a_{\beta},
$$

where the eigenvalues $\omega_{\alpha}$ and $\omega_{\beta}$ of the single excitons are

$$
\begin{aligned}
& \omega_{\alpha}=\omega+\Delta \sec 2 \theta, \\
& \omega_{\beta}=\omega-\Delta \sec 2 \theta .
\end{aligned}
$$

Denoting $|g\rangle$ as the molecular ground state or the excitonic vaccuum, $|A\rangle=a_{A}^{+}|g\rangle$ and $|B\rangle=a_{B}^{+}|g\rangle$ are the excitons at each site, whereas $|\alpha\rangle=a_{\alpha}^{+}|g\rangle,|\beta\rangle=a_{\beta}^{+}|g\rangle$ are the delocalized excitons. The biexcitonic state, expressed by $|f\rangle$ $=a_{A}^{+} a_{B}^{+}|g\rangle=a_{\alpha}^{+} a_{\beta}^{+}|g\rangle$, also plays a role in our study, as it is resonantly accessed through excited state absorption (ESA) after several pulses. Notice that the Hamiltonian $H_{S}$ does not contain two-body operators and, therefore, does not account for exciton-exciton binding or repulsion terms, so the energy level of the biexciton is just the sum of the two exciton energies, $\omega_{f}=\omega_{\alpha}+\omega_{\beta}=\omega_{A}+\omega_{B}{ }^{2}$ Defining $\omega_{i j} \equiv \omega_{i}-\omega_{j}$, the following relations hold:

$$
\begin{aligned}
& \omega_{\alpha g}=\omega_{f \beta}, \\
& \omega_{\beta g}=\omega_{f \alpha} .
\end{aligned}
$$

Since we are concerned with the interaction of the chromophores with electromagnetic radiation, we make some remarks on the geometry of the transition dipoles. Let $\boldsymbol{\mu}_{i j}$ $=\langle i|\hat{\boldsymbol{\mu}}| j\rangle$. Assume that the transition dipole moments from the ground to the single excitons in the site basis are $\mu_{g A}$ $=\boldsymbol{\mu}_{A g}=\boldsymbol{d}_{A}$ and $\boldsymbol{\mu}_{g B}=\boldsymbol{\mu}_{B g}=\boldsymbol{d}_{B}$, respectively. It follows that the dipole moments $\boldsymbol{\mu}_{i j}$ for $i, j \in\{\alpha, \beta, f\}$ are located in the same plane but in general have different magnitudes and directions:

$$
\begin{aligned}
& {\left[\begin{array}{l}
\boldsymbol{\mu}_{\alpha g} \\
\boldsymbol{\mu}_{\beta g}
\end{array}\right]=\left[\begin{array}{cc}
\cos \theta & \sin \theta \\
-\sin \theta & \cos \theta
\end{array}\right]\left[\begin{array}{l}
\boldsymbol{d}_{A} \\
\boldsymbol{d}_{B}
\end{array}\right]} \\
& {\left[\begin{array}{l}
\boldsymbol{\mu}_{f \alpha} \\
\boldsymbol{\mu}_{f \beta}
\end{array}\right]=\left[\begin{array}{cc}
\sin \theta & \cos \theta \\
\cos \theta & -\sin \theta
\end{array}\right]\left[\begin{array}{l}
\boldsymbol{d}_{A} \\
\boldsymbol{d}_{B}
\end{array}\right] .}
\end{aligned}
$$

We also have $\boldsymbol{\mu}_{i j}=\boldsymbol{\mu}_{j i}$. As enumerated in our model, dipole mediated transitions only couple the ground state to the single excitons and the single excitons to the biexciton.

\section{PHOTON-ECHO EXPERIMENT AS QUANTUM PROCESS TOMOGRAPHY}

In a four-wave mixing experiment, an ensemble of identical dimers interact with a series of three ultrashort laser 
pulses. The perturbation due to these pulses is given by

$$
V(\boldsymbol{r}, t)=-\lambda \sum_{i=1}^{3} \hat{\boldsymbol{\mu}} \cdot \boldsymbol{e}_{i} E\left(t-t_{i}\right) e^{i \boldsymbol{k}_{i} \cdot \boldsymbol{r}-i \omega_{i}\left(t-t_{i}\right)}+c . c .,
$$

where $\lambda$ is the intensity of the electric field, which is assumed to be weak compared to the characteristic energy scales of $H_{S}, \hat{\boldsymbol{\mu}}$ is the dipole operator, $\boldsymbol{e}_{\boldsymbol{i}}, t_{i}, \boldsymbol{k}_{i}, \omega_{i}$ denote the polarization, ${ }^{119}$ time center, wavevector, and carrier frequency of the $i t h$ pulse, and $\boldsymbol{r}$ is the position of the center of mass of the molecule. $E(t)$ is the slowly varying pulse envelope, which we choose as a Gaussian with width $\sigma$, or full-width half-maximum $F W H M=2 \sqrt{2 \ln 2} \sigma, E(t)=e^{-t^{2} /\left(2 \sigma^{2}\right)}$. The pulses are sent to the sample in a noncollinear fashion, generating a time-dependent dipole in each of the molecules. Since the characteristic size of a molecule is much smaller than the wavelength of the radiation, $2 \pi /\left|\boldsymbol{k}_{i}\right|$, each molecule only experiences a potential that changes in time but is uniform in space, consistent with the dipole approximation. Nonetheless, the spatial dependence of the pulses is still important, as the phases $e^{ \pm i \boldsymbol{k}_{i} \cdot \boldsymbol{r}}$ are imprinted to molecules located across different positions $\boldsymbol{r}$ in the sample. The size of the sample is much larger than $2 \pi /\left|\boldsymbol{k}_{i}\right|$, so there is a considerable spacial modulation of the polarization due to these phases. Denoting the time-dependent state of the molecule at position $\boldsymbol{r}$ by $\rho(\boldsymbol{r}, t)$, a perturbative treatment allows us to decompose the density matrix into Fourier components:

$$
\rho(\boldsymbol{r}, t)=\sum_{s} \rho_{s}(t) e^{i \boldsymbol{k}_{s} \cdot \boldsymbol{r}},
$$

where $\boldsymbol{k}_{s}=l \boldsymbol{k}_{1}+m \boldsymbol{k}_{2}+n \boldsymbol{k}_{3}$ and $l, n, m$ are integers. Notice that $\boldsymbol{k}_{s}$ equals a linear combination of the wavevectors associated with each pulse. Depending on the location of the molecule, each pulse will act with a spatial phase, so that the total phase accumulated in the quantum state of the molecule at $\boldsymbol{r}$ equals $e^{i \boldsymbol{k}_{s} \cdot \boldsymbol{r}}$ for a given combination of perturbations. Each improper density matrix $\rho_{s}(t)$ corresponds to one of these phases and can be calculated by keeping track of the actions of the pulses in the bra and the ket of the system using double-sided Feynman diagrams. ${ }^{1,2}$ Equation (12) implies that the optical polarization induced on the molecule can also be Fourier decomposed into different components: ${ }^{61-63}$ $\boldsymbol{P}(\boldsymbol{r}, t)=\operatorname{Tr}(\hat{\boldsymbol{\mu}}(\boldsymbol{r}) \rho(\boldsymbol{r}, t))=\sum_{s} \boldsymbol{P}_{s}(t) e^{i \boldsymbol{k}_{s} \cdot \boldsymbol{r}}$, where $\hat{\boldsymbol{\mu}}(\boldsymbol{r})$ denotes the dipole operator of the molecule located at $\boldsymbol{r}$. The experimental setting we describe is analogous to the one of an array of dipole antennas which are spatially phased in a grating with respect to each other and oscillate in time. Classical electromagnetism predicts that the induced macroscopic polarization of this array emits radiation which is precisely concentrated along the vectors $\boldsymbol{k}_{s}$. This condition, which reflects conservation of momentum of the fields, is known as phase matching. ${ }^{64} \mathrm{~A}$ fourth pulse of the same wavevector as one of the $\boldsymbol{k}_{s}$, known as the local oscillator, is allowed to interfere with the radiation along that direction. By varying the phases of this fourth field, two heterodyne detections can be carried out to extract the real and imaginary components of $P_{s}(t) \equiv \boldsymbol{P}_{s}(t) \cdot \boldsymbol{e}_{4}$, respectively, where $\boldsymbol{e}_{4}$ is the polarization of the local oscillator.
In this article, we are interested in the signal along $\boldsymbol{k}_{P E}$ $=-\boldsymbol{k}_{1}+\boldsymbol{k}_{2}+\boldsymbol{k}_{\mathbf{3}}$, the so called PE direction. ${ }^{65}$ The frequency components of the pulses lie within the optical regime, so they can induce the transitions enumerated in Sec. III. Traditionally, in the MDOS literature, the intervals between the time centers of the pulses are called coherence $\tau=t_{2}-t_{1}$, waiting $T=t_{3}-t_{2}$, and echo $t=t_{4}-t_{3}$ times, respectively. Here, $t_{4}$ is the time of detection of the signal. ${ }^{66}$ We shall only consider rephasing photon-echo signals, where $t_{1}<t_{2}<t_{3}<t_{4}$, where the inhomogeneous broadening is rephased. ${ }^{67}$ Due to these explicit interval dependences, the collected signal can be expressed as $P_{P E}(\tau, T, t)$.

As explained in our previous studies ${ }^{42,116}$ the PE experiment for a dimer probes its single exciton dynamics, and the data resulting from a set of experiments with different controls yield a QPT. In fact, the polarization signal for this system may be expressed as a linear combination of elements of the process matrix $\chi(T)$ :

$$
\left[P_{P E}\right]_{\boldsymbol{e}_{1}, \boldsymbol{e}_{2}, \boldsymbol{e}_{3}, \boldsymbol{e}_{4}}^{\omega_{1}, \omega_{2}, \omega_{3}}(t)=\sum_{p, q, r} C_{\omega_{1}}^{p} C_{\omega_{2}}^{q} C_{\omega_{3}}^{r} P_{\boldsymbol{e}_{1}, \boldsymbol{e}_{2}, \boldsymbol{e}_{3}, \boldsymbol{e}_{4}}^{p, q}(t),
$$

where,

$$
\begin{aligned}
& P_{\boldsymbol{e}_{1}, \boldsymbol{e}_{2}, \boldsymbol{e}_{3}, \boldsymbol{e}_{4}}^{p, q}(t) \\
= & -\left(\boldsymbol{\mu}_{p g} \cdot \boldsymbol{e}_{1}\right)\left(\boldsymbol{\mu}_{q g} \cdot \boldsymbol{e}_{2}\right) \mathcal{G}_{g p}(\tau) \\
& \times\left\{\left[\left(\boldsymbol{\mu}_{\alpha g} \cdot \boldsymbol{e}_{3}\right)\left(\boldsymbol{\mu}_{\alpha g} \cdot \boldsymbol{e}_{4}\right) \mathcal{G}_{\alpha g}(t)\right.\right. \\
& \times\left(\chi_{g g q p}(T)-\delta_{p q}-\chi_{\alpha \alpha q p}(T)\right) \\
& +\left(\boldsymbol{\mu}_{f \beta} \cdot \boldsymbol{e}_{3}\right)\left(\boldsymbol{\mu}_{f \beta} \cdot \boldsymbol{e}_{4}\right) \mathcal{G}_{f \beta}(t) \chi_{\beta \beta q p}(T) \\
& +\left(\left(\boldsymbol{\mu}_{f \beta} \cdot \boldsymbol{e}_{3}\right)\left(\boldsymbol{\mu}_{f \alpha} \cdot \boldsymbol{e}_{4}\right) \mathcal{G}_{f \alpha}(t)\right. \\
& \left.\left.\left.-\left(\boldsymbol{\mu}_{\alpha g} \cdot \boldsymbol{e}_{3}\right)\left(\boldsymbol{\mu}_{\beta g} \cdot \boldsymbol{e}_{4}\right) \mathcal{G}_{\beta g}(t)\right) \chi_{\beta \alpha q p}(T)\right]\right\},
\end{aligned}
$$

and the analogous expression for $P_{\boldsymbol{e}_{1}, \boldsymbol{e}_{2}, \boldsymbol{e}_{3}, \boldsymbol{e}_{4}}^{p, q, \boldsymbol{e}_{2}}(t)$ follows by carrying out the substitutions $\{\alpha, \beta\} \rightarrow\{\beta, \alpha\}$ in Eq. (14).

The coefficients $C_{\omega_{i}}^{p}$ for $p \in\{\alpha, \beta\}$ are frequency amplitudes of the laser pulse which is centered at $\omega_{i}$, evaluated at the transition energy $\omega_{p g}$ :

$$
C_{\omega_{i}}^{p}=-\frac{\lambda}{i} \sqrt{2 \pi \sigma^{2}} e^{-\sigma^{2}\left(\omega_{p g}-\omega_{i}\right)^{2} / 2},
$$

and

$$
\mathcal{G}_{i j}(\tau)=\Theta(\tau) e^{\left(-i \omega_{i j}-\Gamma_{i j}\right) \tau}
$$

is the propagator of the optical coherences $|i\rangle\langle j|$ in the coherence and echo times, which, has been taken to be the product of a coherent oscillatory term beating at a frequency $\omega_{i j}$ and an exponential decay with dephasing rate $\Gamma_{i j}$. This propagator is defined only for $\tau>0$ via the step function $\Theta(\tau)$. The frequencies of the coherences in the coherence and echo intervals have opposite signs, reflecting the rephasing character of the signal. In optical PE experiments, it is customary to assume that the free-induction decay characterized by the evolution of optical coherences in the coherence and echo times is well characterized and given by expressions of the form of Eq. (16). The reason is that the characteristic energetic scales of the vibrational degrees of freedom are much lower than the optical gap, so the only nonunitary dynamics they induce in the optical coherence is, to a good approximation, restricted 
to dephasing $\Gamma_{i j}$ which can be inferred from the polarization signal. ${ }^{120}$ The dynamics in the waiting time is more complex, consisting of small frequencies due to excitonic superpositions which are strongly influenced by the bath. It is the latter interval where QPT will prove useful.

The polarization signal yields a linear combination of elements $\chi_{a b c d}(T)$ weighted by the probability amplitude to prepare a state $|c\rangle\langle d|$ with the first two pulses and detect $|a\rangle\langle b|$ with the third pulse and the fourth heterodyning pulse. These probability amplitudes can be controlled by manipulating the polarization of the pulses $\boldsymbol{e}_{i}$ as well as the frequency amplitudes for the resonant transitions $C_{\omega_{1}}^{p}, C_{\omega_{2}}^{q}, C_{\omega_{3}}^{r}$. In essence, state preparation and QST are implicit in the coherence and echo times, respectively (see Ref. 42). In a different context, Gelin and Kosov had previously hinted at a similar idea by identifying these times as "doorway" and "window" intervals. ${ }^{68}$ By conducting several experiments varying these control knobs and collecting the signal from each of these settings, a system of linear equations can be established whereby the elements of $\chi(T)$ can be inverted, and therefore QPT is achieved. This statement is correct provided that besides the free-induction decay rates $\Gamma_{i j}$, the parameters $\omega_{\alpha g}$, $\omega_{\beta g}, \boldsymbol{\mu}_{\alpha g}, \boldsymbol{\mu}_{\beta g}, \boldsymbol{\mu}_{f \alpha}$, and $\boldsymbol{\mu}_{f \beta}$ are all known or can be obtained self-consistently during the experiment. We will elaborate on these points for the case of a homodimer in Sec. V.

Notice that Eq. (13) monitors all the 12 real valued parameters involving $\chi_{a b c d}(T)$ for $a, b, c, d \in\{\alpha, \beta\}$, so that they allow for the QPT of the single-exciton manifold, which is an effective quantum bit (qubit) system. However, it also keeps track of the elements $\chi_{g g c d}(T) c, d \in\{\alpha, \beta\}$, that is, the possibility of amplitude leakage errors from the single-exciton channel to $|g\rangle\langle g|$. It is known that whereas the excitonic dynamics occurs in femtosecond time scales, exciton recombination happens in the order of nanoseconds. Therefore, these decay channels could be potentially ignored in many experimental systems. We shall keep them in our theoretical analysis as they do not increase the complexity of the problem by much, although in situations where this could be problematic, we could accordingly disregard them.

\section{QPT FROM 2D SPECTRUM OF PE}

As mentioned, QPT can be carried out from data resulting from a series of experiments varying colors and polarizations of the pulses. The necessary information can in principle be obtained by collecting a single point datum for a fixed pair of $\tau$ and $t$ points for each of the experiments. Often, however, the PE signal is collected across many $\tau, T, t$ points, and conveniently processed into a $2 \mathrm{D}$ correlation spectrum in the conjugate frequency variables $\omega_{\tau}$ and $\omega_{t}$ :

$$
S\left(\omega_{\tau}, T, \omega_{t}\right)=i \int_{0}^{\infty} d \tau e^{-i \omega_{\tau} \tau} \int_{0}^{\infty} d t e^{i \omega_{t} t} P_{P E}(\tau, T, t),
$$

which still evolves in the $T$ coordinate. ${ }^{121}$ By performing the integrals of Eq. (17) using Eq. (13), we obtain:

$$
S\left(\omega_{\tau}, T, \omega_{t}\right)=i \sum_{m, n=\alpha, \beta} l_{\tau, m}\left(\omega_{\tau}\right) l_{t, n}\left(\omega_{t}\right) S_{m n}(T) .
$$

The spectrum consists of a sum of four resonances at $\left(\omega_{\tau}\right.$, $\left.\omega_{t}\right) \in\left\{\left(\omega_{\alpha g}, \omega_{\alpha g}\right),\left(\omega_{\alpha g}, \omega_{\beta g}\right),\left(\omega_{\beta g}, \omega_{\alpha g}\right),\left(\omega_{\beta g}, \omega_{\beta g}\right)\right\}$, which correspond to the frequencies of the optical coherences at the coherence and echo times. These resonances are modulated by line shape functions of the form

$$
\begin{aligned}
& l_{\tau, m}\left(\omega_{\tau}\right)=\frac{1}{i\left(\omega_{\tau}-\omega_{m g}-i \Gamma_{m g}\right)}, \\
& l_{t, n}\left(\omega_{t}\right)=\frac{1}{i\left(-\omega_{t}+\omega_{n g}-i \Gamma_{n g}\right)},
\end{aligned}
$$

that correspond to the one-sided Fourier transform of the propagator along each $\tau$ and $t$ axis. ${ }^{122}$ The peaks are centered about $\omega=\omega_{m g}$ and have a width parameter $\Gamma_{m g}$. The difference in signs for the Fourier transform in Eq. (17) guarantees that all the resonances appear in the first quadrant of both frequency axes. The expressions for the amplitudes $S_{m n}(T)$, associated with peaks centered at $\left(\omega_{\tau}, \omega_{t}\right)=\left(\omega_{m g}, \omega_{n g}\right)$, are given by ${ }^{123}$

$$
\begin{aligned}
S_{\alpha \alpha}(T)= & -i C_{\omega_{1}}^{\alpha} C_{\omega_{2}}^{\alpha}\left(\boldsymbol{\mu}_{\alpha g} \cdot \boldsymbol{e}_{1}\right)\left(\boldsymbol{\mu}_{\alpha g} \cdot \boldsymbol{e}_{2}\right) \\
& \times\left\{C _ { \omega _ { 3 } } ^ { \alpha } \left[\left(\boldsymbol{\mu}_{\alpha g} \cdot \boldsymbol{e}_{3}\right)\left(\boldsymbol{\mu}_{\alpha g} \cdot \boldsymbol{e}_{4}\right)\left(\chi_{g g \alpha \alpha}(T)-1-\chi_{\alpha \alpha \alpha \alpha}(T)\right)\right.\right. \\
& \left.+\left(\boldsymbol{\mu}_{f \beta} \cdot \boldsymbol{e}_{3}\right)\left(\boldsymbol{\mu}_{f \beta} \cdot \boldsymbol{e}_{4}\right) \chi_{\beta \beta \alpha \alpha}(T)\right] \\
& +C_{\omega_{3}}^{\beta}\left[\left(\boldsymbol{\mu}_{f \alpha} \cdot \boldsymbol{e}_{3}\right)\left(\boldsymbol{\mu}_{f \beta} \cdot \boldsymbol{e}_{4}\right)-\left(\boldsymbol{\mu}_{\beta g} \cdot \boldsymbol{e}_{3}\right)\right. \\
& \left.\left.\left.\times\left(\boldsymbol{\mu}_{\alpha g} \cdot \boldsymbol{e}_{4}\right)\right) \chi_{\alpha \beta \alpha \alpha}(T)\right]\right\} \\
& -i C_{\omega_{1}}^{\alpha} C_{\omega_{2}}^{\beta}\left(\boldsymbol{\mu}_{\alpha g} \cdot \boldsymbol{e}_{1}\right)\left(\boldsymbol{\mu}_{\beta g} \cdot \boldsymbol{e}_{2}\right) \\
& \times\left\{C _ { \omega _ { 3 } } ^ { \alpha } \left[\left(\boldsymbol{\mu}_{\alpha g} \cdot \boldsymbol{e}_{3}\right)\left(\boldsymbol{\mu}_{\alpha g} \cdot \boldsymbol{e}_{4}\right)\left(\chi_{g g \beta \alpha}(T)-\chi_{\alpha \alpha \beta \alpha}(T)\right)\right.\right. \\
& \left.+\left(\boldsymbol{\mu}_{f \beta} \cdot \boldsymbol{e}_{3}\right)\left(\boldsymbol{\mu}_{f \beta} \cdot \boldsymbol{e}_{4}\right) \chi_{\beta \beta \beta \alpha}(T)\right] \\
& +C_{\omega_{3}}^{\beta}\left[\left(\left(\boldsymbol{\mu}_{f \alpha} \cdot \boldsymbol{e}_{3}\right)\left(\boldsymbol{\mu}_{f \beta} \cdot \boldsymbol{e}_{4}\right)-\left(\boldsymbol{\mu}_{\beta g} \cdot \boldsymbol{e}_{3}\right)\right.\right. \\
& \left.\left.\left.\times\left(\boldsymbol{\mu}_{\alpha g} \cdot \boldsymbol{e}_{4}\right)\right) \chi_{\alpha \beta \beta \alpha}(T)\right]\right\},
\end{aligned}
$$

$$
\begin{aligned}
S_{\alpha \beta}(T)= & -i C_{\omega_{1}}^{\alpha} C_{\omega_{2}}^{\alpha}\left(\boldsymbol{\mu}_{\alpha g} \cdot \boldsymbol{e}_{1}\right)\left(\boldsymbol{\mu}_{\alpha g} \cdot \boldsymbol{e}_{2}\right) \\
& \times\left\{C _ { \omega _ { 3 } } ^ { \beta } \left[\left(\boldsymbol{\mu}_{\beta g} \cdot \boldsymbol{e}_{3}\right)\left(\boldsymbol{\mu}_{\beta g} \cdot \boldsymbol{e}_{4}\right)\left(\chi_{g g \alpha \alpha}(T)-1-\chi_{\beta \beta \alpha \alpha}(T)\right)\right.\right. \\
& \left.+\left(\boldsymbol{\mu}_{f \alpha} \cdot \boldsymbol{e}_{3}\right)\left(\boldsymbol{\mu}_{f \alpha} \cdot \boldsymbol{e}_{4}\right) \chi_{\alpha \alpha \alpha \alpha}(T)\right] \\
& +C_{\omega_{3}}^{\alpha}\left[\left(\left(\boldsymbol{\mu}_{f \beta} \cdot \boldsymbol{e}_{3}\right)\left(\boldsymbol{\mu}_{f \alpha} \cdot \boldsymbol{e}_{4}\right)-\left(\boldsymbol{\mu}_{\alpha g} \cdot \boldsymbol{e}_{3}\right)\right.\right. \\
& \left.\left.\left.\times\left(\boldsymbol{\mu}_{\beta g} \cdot \boldsymbol{e}_{4}\right)\right) \chi_{\beta \alpha \alpha \alpha}(T)\right]\right\} \\
& -i C_{\omega_{1}}^{\alpha} C_{\omega_{2}}^{\beta}\left(\boldsymbol{\mu}_{\alpha g} \cdot \boldsymbol{e}_{1}\right)\left(\boldsymbol{\mu}_{\beta g} \cdot \boldsymbol{e}_{2}\right) \\
& \times\left\{C _ { \omega _ { 3 } } ^ { \beta } \left[\left(\boldsymbol{\mu}_{\beta g} \cdot \boldsymbol{e}_{3}\right)\left(\boldsymbol{\mu}_{\beta g} \cdot \boldsymbol{e}_{4}\right)\left(\chi_{g g \beta \alpha}(T)-\chi_{\beta \beta \beta \alpha}(T)\right)\right.\right. \\
& \left.+\left(\boldsymbol{\mu}_{f \alpha} \cdot \boldsymbol{e}_{3}\right)\left(\boldsymbol{\mu}_{f \alpha} \cdot \boldsymbol{e}_{4}\right) \chi_{\alpha \alpha \beta \alpha}(T)\right] \\
& +C_{\omega_{3}}^{\alpha}\left[\left(\left(\boldsymbol{\mu}_{f \beta} \cdot \boldsymbol{e}_{3}\right)\left(\boldsymbol{\mu}_{f \alpha} \cdot \boldsymbol{e}_{4}\right)-\left(\boldsymbol{\mu}_{\alpha g} \cdot \boldsymbol{e}_{3}\right)\right.\right. \\
& \left.\left.\left.\times\left(\boldsymbol{\mu}_{\beta g} \cdot \boldsymbol{e}_{4}\right)\right) \chi_{\beta \alpha \beta \alpha}(T)\right]\right\},
\end{aligned}
$$

and $S_{\beta \beta}(T), S_{\beta \alpha}(T)$ follow immediately from Eqs. (21) and (22) by performing the substitutions $\{\alpha, \beta\} \rightarrow\{\beta, \alpha\}$. Typically, the probed samples are in solution, so the molecules in the ensemble are isotropically distributed. The isotropic average $\langle\cdot\rangle$ for a tetradic $\left(\boldsymbol{\mu}_{a} \cdot \boldsymbol{e}_{1}\right)\left(\boldsymbol{\mu}_{b} \cdot \boldsymbol{e}_{2}\right)\left(\boldsymbol{\mu}_{c} \cdot \boldsymbol{e}_{3}\right)\left(\boldsymbol{\mu}_{d} \cdot \boldsymbol{e}_{4}\right)$ is 

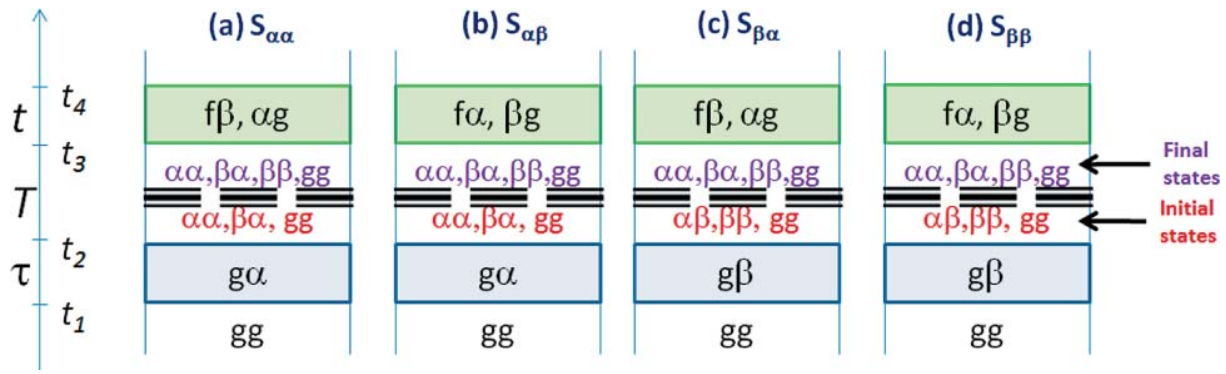

FIG. 1. Liouville space pathways corresponding to each of the four resonances in the rephasing 2D-ES of a coupled dimer. The amplitude $S_{m n}(T)$ corresponds to the peak located at $\left(\omega_{\tau}, \omega_{t}\right)=\left(\omega_{m g}, \omega_{n g}\right)$, which are the values of the optical frequencies at the coherence and echo time intervals $\tau$ and $t$, respectively. These amplitudes provide information on the coherent and incoherent excitonic processes at the waiting time $T$ by enumerating the possible initial and final states at the waiting time $T$ which satisfy the PE phase matching condition for the pulses acting at times $t_{1}, t_{2}, t_{3}, t_{4}$. The information contained in the amplitudes $S_{m n}(T)$ can be distilled to reconstruct the process matrix for the single-exciton manifold of the dimer, thus allowing a QPT.

given by ${ }^{69}$

$$
\begin{aligned}
& \left\langle\left(\boldsymbol{\mu}_{a} \cdot \boldsymbol{e}_{1}\right)\left(\boldsymbol{\mu}_{b} \cdot \boldsymbol{e}_{2}\right)\left(\boldsymbol{\mu}_{c} \cdot \boldsymbol{e}_{3}\right)\left(\boldsymbol{\mu}_{d} \cdot \boldsymbol{e}_{4}\right)\right\rangle \\
& \quad=\sum_{m_{1} m_{2} m_{3} m_{4}} I_{e_{1} e_{2} e_{3} e_{4} ; m_{1} m_{2} m_{3} m_{4}}^{(4)} \\
& \quad \times\left[\left(\boldsymbol{\mu}_{a} \cdot \boldsymbol{m}_{1}\right)\left(\boldsymbol{\mu}_{b} \cdot \boldsymbol{m}_{2}\right)\left(\boldsymbol{\mu}_{c} \cdot \boldsymbol{m}_{3}\right)\left(\boldsymbol{\mu}_{d} \cdot \boldsymbol{m}_{4}\right)\right],
\end{aligned}
$$

$$
\begin{aligned}
& I_{e_{1} e_{2} e_{3} e_{4} ; m_{1} m_{2} m_{3} m_{4}} \\
& \quad=\frac{1}{30}\left[\delta_{e_{1} e_{2}} \delta_{e_{3} e_{4}} \delta_{e_{1} e_{3}} \delta_{e_{2} e_{4}} \delta_{e_{1} e_{4}} \delta_{e_{2} e_{3}}\right] \\
& \quad \times\left[\begin{array}{ccc}
4 & -1 & -1 \\
-1 & 4 & -1 \\
-1 & -1 & 4
\end{array}\right]\left[\begin{array}{l}
\delta_{m_{1} m_{2}} \delta_{m_{3} m_{4}} \\
\delta_{m_{1} m_{3}} \delta_{m_{2} m_{4}} \\
\delta_{m_{1} m_{4}} \delta_{m_{2} m_{3}}
\end{array}\right],
\end{aligned}
$$

where $\boldsymbol{e}_{i}$ and $\boldsymbol{m}_{i}$ are the polarizations of the pulses in the lab and the molecular frames, respectively. The isotropic average consists of a sum of molecular frame products $\left[\left(\boldsymbol{\mu}_{a} \cdot \boldsymbol{m}_{1}\right)\left(\boldsymbol{\mu}_{b}\right.\right.$. $\left.\left.\boldsymbol{m}_{2}\right)\left(\boldsymbol{\mu}_{c} \cdot \boldsymbol{m}_{3}\right)\left(\boldsymbol{\mu}_{d} \cdot \boldsymbol{m}_{4}\right)\right]$ weighted by the isotropically invariant tensor $I_{e_{1} e_{2} e_{3} e_{4} ; m_{1} m_{2} m_{3} m_{4}}^{(4)}$.

Since the information in Eq. (18) is in principle contained in Eq. (13), several conclusions from our previous study are immediately transferable: The elements of $\chi(T)$ can be all be extracted by repeating a number of experiments with different polarization configurations for the fields and two different waveforms for the pulses. Under different motivations, theoretical proposals for manipulating 2D-ES using pulse-shaping capabilities have been previously reported. ${ }^{70,71}$ An extensive study of this possibility for a heterodimer will be presented in the second article accompanying this study.

Equations (21), (22), and their analogues upon the $\{\alpha, \beta\} \rightarrow\{\beta, \alpha\}$ substitutions can also be derived by classifying the double-sided Feynman diagrams that oscillate at the particular frequencies for the coherence and waiting times in each of the four resonances (Fig. 1). In analyzing the possible pathways in Liouville space, we make use of the rotating wave approximation: Perturbations which are proportional to $-e^{-i \boldsymbol{k}_{i} \cdot \boldsymbol{r}+i \omega_{i}\left(t-t_{i}\right)} \hat{\boldsymbol{\mu}} \cdot \boldsymbol{e}_{i}$ can de-excite the ket and excite the bra, whereas the ones proportional to $-e^{i \boldsymbol{k}_{i} \cdot \boldsymbol{r}-i \omega_{i}\left(t-t_{i}\right)} \hat{\boldsymbol{\mu}} \cdot \boldsymbol{e}_{i}$ can excite the ket and de-excite the bra. As an illustration, consider the signal $S_{\alpha \beta}(T)$, which arises from diagrams oscillating with frequency $\omega_{g \alpha}$ at the coherence time and $\omega_{\beta g}$ at the echo time [Fig. 1(b)]. The two states at the coherence time which can oscillate at $\omega_{g \alpha}$ are $|g\rangle\langle\alpha|$ or $|\beta\rangle\langle f|$, but the latter cannot be produced by a single action of the dipole operator on the initial ground state $|g\rangle\langle g|$. Therefore, $|g\rangle\langle\alpha|$ is the only possible state for the coherence interval and is produced by acting the first pulse on the bra of the ground state: $|g\rangle\langle g|\rightarrow| g\rangle\langle\alpha|$. Similar considerations imply that the state at the echo time must be $|\beta\rangle\langle g|$ or $|f\rangle\langle\alpha|$. Given these constraints, we are ready to enumerate the possible initial and final states for the waiting time interval which are compatible with these restrictions. By exciting the ket or deexciting the bra of $|g\rangle\langle\alpha|$ with the second pulse, the following initial states $|c\rangle\langle d|$ for the quantum channel can be produced: $|c\rangle\langle d| \in\{|\alpha\rangle\langle\alpha|,| \beta\rangle\langle\alpha|| g\rangle,\langle g|\}$. The final states $|a\rangle\langle b| \in\{|\alpha\rangle\langle\alpha|,| \beta\rangle\langle\alpha|,| \beta\rangle\langle\beta|| g\rangle,\langle g|\}$ can all give rise to $|\beta\rangle\langle g|$ or $|f\rangle\langle\alpha|$ by exciting the ket or de-exciting the bra with the third pulse. Therefore, in principle, there are $4 \times 3=12$ possibilities for $\chi_{a b c d}(T)$ which contribute to $S_{\alpha \beta}(T)$. However, we assume that the state $|g\rangle\langle g|$ does not evolve to other states due to the bath:

$$
\chi_{a b g g}(T)=\delta_{a g} \delta_{b g},
$$

This assumption is quite reasonable, as we are ignoring processes where phonons can induce optical excitations from $|g\rangle\langle g|$. This condition is present in Eq. (14) in the $\delta$-function terms and in Eqs. (21) and (22) in the " -1 " terms, which correspond to $-\chi_{g g g g}(T)$. This leaves $12-4=8$ possibilities for $\chi_{a b c d}(T)$ contributing to $S_{\alpha \beta}(T)$.

To be more explicit, consider the pathways in $S_{\alpha \beta}(T)$ that monitor the population to coherence process $\chi_{\beta \alpha \alpha \alpha}(T)$. These are displayed in Fig. (2). The pathway on the left represents
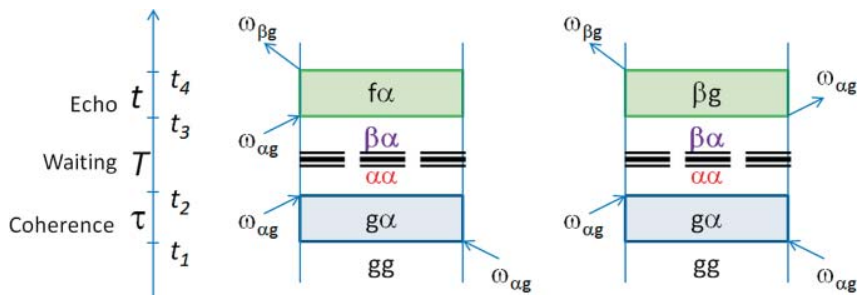

FIG. 2. A more detailed view on the Liouville space pathways corresponding to the monitoring of the population to coherence process $\chi_{\beta \alpha \alpha \alpha}(T)$ in the peak at $\left(\omega_{\tau}, \omega_{t}\right)=\left(\omega_{\alpha g}, \omega_{\beta g}\right)$ of the rephasing 2D-ES. These diagrams belong to the amplitude $S_{\alpha \beta}(T)$ and can be easily constructed by taking into account the PE phase matching and the resonant conditions. 


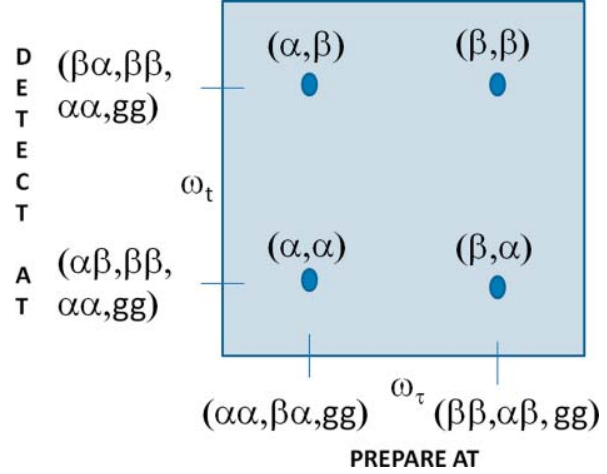

FIG. 3. Summary of QPT for a coupled dimer in the rephasing 2D-ES. The Liouville pathways depicted in Fig. 1 can be condensed into this diagram. The horizontal axis for the coherence frequency $\omega_{\tau}$ is associated with a state preparation, whereas the vertical axis for the echo frequency $\omega_{t}$ corresponds to a detection. The four resonances labeled as $(m, n)$ correspond to peaks located at $\left(\omega_{\tau}, \omega_{t}\right)=\left(\omega_{m g}, \omega_{n g}\right)$. Their amplitudes contain information on $\chi_{a b c d}(T)$, where $c d$ is the state prepared at the beginning of the waiting time interval and $a b$ the state detected at the end of the same interval. For instance, the peak at $\left(\omega_{\alpha}, \omega_{\beta}\right)$ keeps track of the elements $\chi_{a b c d}(T)$ where $|c\rangle\langle d|$ $\in\{|\alpha\rangle\langle\alpha|,| \beta\rangle\langle\alpha|| g\rangle,\langle g|\}$ and $|a\rangle\langle b| \in\{|\beta\rangle\langle\alpha|,| \beta\rangle\langle\beta|,| \alpha\rangle\langle\alpha|| g\rangle,\langle g|\}$.

the ESA from the single-exciton manifold and is proportional to $\left(-C_{\omega_{1}}^{\alpha} \boldsymbol{\mu}_{\alpha g} \cdot \boldsymbol{e}_{1}\right)\left(C_{\omega_{2}}^{\alpha} \boldsymbol{\mu}_{\alpha g} \cdot \boldsymbol{e}_{2}\right)\left(C_{\omega_{3}}^{\alpha} \boldsymbol{\mu}_{f \beta} \cdot \boldsymbol{e}_{3}\right)\left(\boldsymbol{\mu}_{f \alpha} \cdot \boldsymbol{e}_{4}\right)$, an expression which can be immediately read out from the diagram: Each interaction with the field picks up a factor corresponding to the amplitude of the transition, which depends on the alignment of the corresponding dipole with the polarization of the pulse, as well as the frequency amplitude of the pulse at the given transition. A minus sign is included if the perturbation is on the bra. Similarly, the pathway on the right involves stimulated emission (SE) and is proportional to $\left(-C_{\omega_{1}}^{\alpha} \boldsymbol{\mu}_{\alpha g} \cdot \boldsymbol{e}_{1}\right)\left(C_{\omega_{2}}^{\alpha} \boldsymbol{\mu}_{\alpha g} \cdot \boldsymbol{e}_{2}\right)\left(-C_{\omega_{3}}^{\alpha} \boldsymbol{\mu}_{\alpha g} \cdot \boldsymbol{e}_{3}\right)\left(\boldsymbol{\mu}_{\beta g} \cdot \boldsymbol{e}_{4}\right)$.

The rest of the diagrams for all the peaks can be systematically analyzed in the way described above. In general, the pathways we need to consider can be classified in ESA, SE, and ground state bleaching (GSB) processes. GSB processes are the ones that take $|g\rangle\langle g|$ at the end of the waiting time to a dipole active coherence involving an excited state and are associated with the $\delta$-function and -1 terms mentioned two paragraphs above. ESA pathways, which are proportional to dipole transitions involving the excited state, differ in sign from SE and GSB pathways, as can be easily noted by inspection.

Figure 3 provides a mnemonic device to keep track of the Liouville pathways that each peak in the $2 \mathrm{D}$ electronic spectrum monitors, and therefore, also provides a scheme for the QPT protocol. The $\omega_{\tau}$ axis can be associated with a particular state preparation, whereas the $\omega_{t}$ axis with a particular detection. Each peak reflects a nontrivial number of processes in Liouville space. As an illustration, we consider in Fig. 4 the ideal case where the bath does not interact with the system, in which case a very simple picture is recovered: the off-diagonal peaks beat at the coherence frequency and the diagonals remain static. This case can be easily derived from the expressions for the peak amplitudes such as Eqs. (21) and (22), by substituting $\chi_{a b c d}(T)=\delta_{a c} \delta_{b d} e^{-i \omega_{a b} T}$, that is, the case where populations remain static, whereas coherences simply beat at exciton difference frequencies.

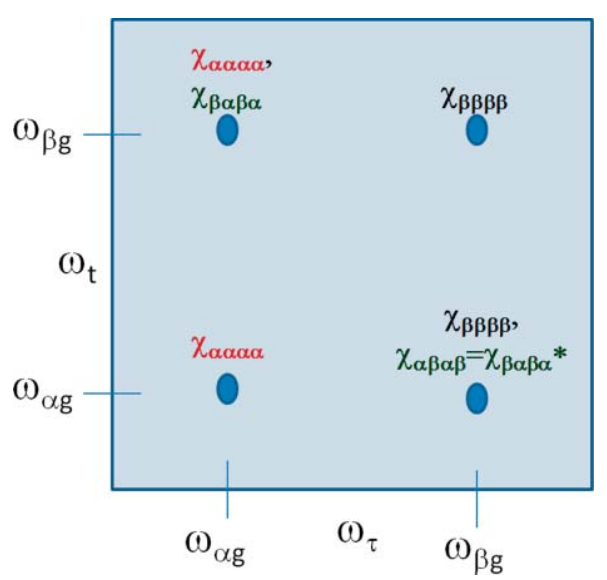

FIG. 4. Rephasing 2D-ES of a coupled dimer in the absence of interactions with a bath. Under unitary dynamics of the excitonic system, each of the four resonances keep track of the elements of $\chi(T)$ indicated in the diagram. Notice that diagonal peaks do not oscillate as a function of waiting time $T$, whereas off-diagonals beat at the frequency equal to the difference in energies of the single exciton eigenstates.

\section{THE CASE OF THE HOMODIMER}

To gain insights into the described QPT protocol, we specialize the results above to a coupled homodimer. In the following subsections, we discuss, for this particular case, (a) the Hamiltonian and the transition dipole moments involved in the experiments, (b) properties of the spectroscopic signals under isotropic averaging, (c) stability of the numerical inversion, (d) analytical expressions of the elements of $\chi(T)$ in terms of the peak amplitudes of the spectra, (e) a procedure to extract the angle $\phi$ between the dipoles, (f) a summary of the QPT procedure, and (g) a numerical example with a model system. A similar study focused on the heterodimer will be presented in the second article of this series.

\section{A. Hamiltonian and transition dipole moments}

In the homodimer, the two sites are identical chromophores with energies $\omega_{A}=\omega_{B}=\bar{\omega}$, and the Hamiltonian in Eqs. (5) and (7) is given by

$$
\begin{aligned}
H_{S} & =\bar{\omega}\left(a_{A}^{+} a_{A}+a_{B}^{+} a_{B}\right)+J\left(a_{A}^{+} a_{B}+a_{B}^{+} a_{A}\right) \\
& =(\bar{\omega}-J) a_{\alpha}^{+} a_{\alpha}+(\bar{\omega}+J) a_{\beta}^{+} a_{\beta},
\end{aligned}
$$

which we have diagonalized with the symmetric $a_{\alpha}^{+}|g\rangle$ and antisymmetric $a_{\beta}^{+}|g\rangle$ single-exciton states given by

$$
\begin{aligned}
& a_{\alpha}^{+}=\frac{1}{\sqrt{2}}\left(a_{A}^{+}+a_{B}^{+}\right), \\
& a_{\beta}^{+}=\frac{1}{\sqrt{2}}\left(a_{A}^{+}-a_{B}^{+}\right) .
\end{aligned}
$$

Using Eq. (10), the transition dipoles take the simple forms:

$$
\begin{aligned}
\boldsymbol{\mu}_{\alpha g} & =\frac{1}{\sqrt{2}}\left(\boldsymbol{d}_{A}+\boldsymbol{d}_{B}\right), \\
\boldsymbol{\mu}_{\beta g} & =\frac{1}{\sqrt{2}}\left(\boldsymbol{d}_{A}-\boldsymbol{d}_{B}\right), \\
\boldsymbol{\mu}_{f \alpha} & =\boldsymbol{\mu}_{\alpha g}, \\
\boldsymbol{\mu}_{f \beta} & =-\boldsymbol{\mu}_{\beta g} .
\end{aligned}
$$




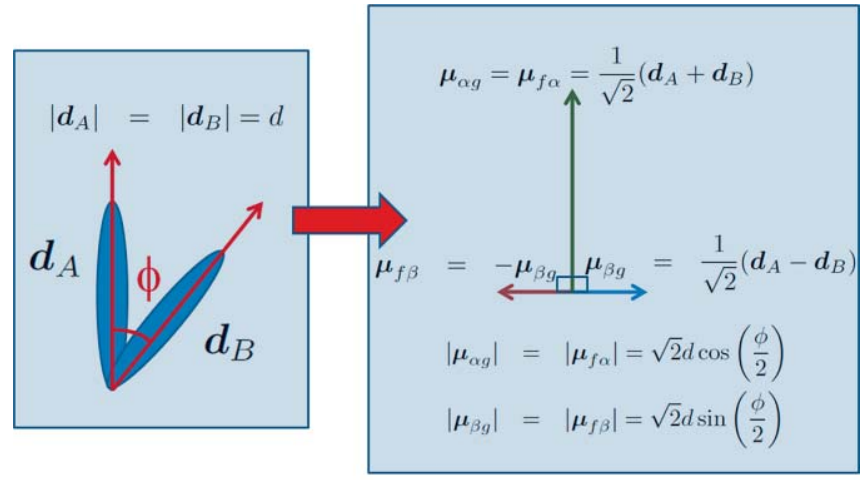

(a)

(b)

FIG. 5. Transition dipole moments of a homodimer. Diagrams for (a) sites and (b) eigenstates.

Interestingly, these expressions are independent of the coupling $J$. Also, notice that $\boldsymbol{\mu}_{\alpha g}$ and $\boldsymbol{\mu}_{f \alpha}$ are perpendicular to $\boldsymbol{\mu}_{\beta g}$ and $\boldsymbol{\mu}_{f \beta}$ (see Fig. 5). Denoting the norm of each site dipole by

$$
\left|\boldsymbol{d}_{A}\right|=\left|\boldsymbol{d}_{B}\right|=d
$$

the following relationships follow:

$$
\begin{aligned}
& \left|\boldsymbol{\mu}_{\alpha g}\right|=\left|\boldsymbol{\mu}_{f \alpha}\right|=\mu_{\alpha g}=\sqrt{2} d \cos \left(\frac{\phi}{2}\right), \\
& \left|\boldsymbol{\mu}_{\beta g}\right|=\left|\boldsymbol{\mu}_{f \beta}\right|=\mu_{\beta g}=\sqrt{2} d \sin \left(\frac{\phi}{2}\right) .
\end{aligned}
$$

As expected, in the degenerate limit that $\phi=0$ or $\pi$ (the site dipoles are parallel or antiparallel), one of the delocalized excitons becomes dark and there is only one bright transition from the ground state. If this is not the case, in general, the two transitions are bright and their dipoles perpendicular to each other. Furthermore, as a difference with the heterodimer case, there are only three (instead of four) different transition dipoles in the homodimer, and two of them are just negative of each other. The degenerate case will be discussed as a limit of the more general one in the next paragraphs.

\section{B. Isotropic averaging of signals}

An important observation regarding isotropic averaging follows:

Claim. Upon isotropic averaging, signals stemming from coherence to population or population to coherence transfer cannot be monitored in the 2D-PE spectrum of a homodimer.

Proof. Without loss of generality, align $\boldsymbol{\mu}_{\beta g}$ and $\boldsymbol{\mu}_{\alpha g}$ in the $\boldsymbol{y}$ and $\boldsymbol{z}$ directions in the frame of the molecule. Equation (23) implies that the only terms in the sum that contribute to an isotropic averaging are the ones where only two or four polarizations of the field are the same. In the expressions for $S_{\alpha \alpha}(T)$ and $S_{\alpha \beta}(T)$ [Eqs. (21) and (22)], as well as the corresponding ones for $S_{\beta \beta}(T)$ and $S_{\beta \alpha}(T)$, all the dipole polarization terms corresponding to coherence to population and the opposite processes involve three dipoles of the same kind and a perpendicular one. Therefore, they vanish under isotropic averaging. As an example, consider the the terms associated with $\chi_{\beta \alpha \alpha \alpha}(T)$ in $S_{\alpha \beta}(T)$ :

$$
\begin{aligned}
& \left\langle\left(-C_{\omega_{1}}^{\alpha} \boldsymbol{\mu}_{\alpha g} \cdot \boldsymbol{e}_{1}\right)\left(C_{\omega_{2}}^{\alpha} \boldsymbol{\mu}_{\alpha g} \cdot \boldsymbol{e}_{2}\right)\left(C_{\omega_{3}}^{\alpha} \boldsymbol{\mu}_{f \beta} \cdot \boldsymbol{e}_{3}\right)\left(\boldsymbol{\mu}_{f \alpha} \cdot \boldsymbol{e}_{4}\right)\right\rangle \\
& \propto\left\langle\left(\boldsymbol{\mu}_{\alpha g} \cdot \boldsymbol{e}_{1}\right)\left(\boldsymbol{\mu}_{\alpha g} \cdot \boldsymbol{e}_{2}\right)\left(\boldsymbol{\mu}_{\beta g} \cdot \boldsymbol{e}_{3}\right)\left(\boldsymbol{\mu}_{\alpha g} \cdot \boldsymbol{e}_{4}\right)\right\rangle_{i s o}=0, \\
& \left\langle\left(-C_{\omega_{1}}^{\alpha} \boldsymbol{\mu}_{\alpha g} \cdot \boldsymbol{e}_{1}\right)\left(C_{\omega_{2}}^{\alpha} \boldsymbol{\mu}_{\alpha g} \cdot \boldsymbol{e}_{2}\right)\left(-C_{\omega_{3}}^{\alpha} \boldsymbol{\mu}_{\alpha g} \cdot \boldsymbol{e}_{3}\right)\left(\boldsymbol{\mu}_{\beta g} \cdot \boldsymbol{e}_{4}\right)\right\rangle \\
& \propto\left\langle\left(\boldsymbol{\mu}_{\alpha g} \cdot \boldsymbol{e}_{1}\right)\left(\boldsymbol{\mu}_{\alpha g} \cdot \boldsymbol{e}_{2}\right)\left(\boldsymbol{\mu}_{\alpha g} \cdot \boldsymbol{e}_{3}\right)\left(\boldsymbol{\mu}_{\beta g} \cdot \boldsymbol{e}_{4}\right)\right\rangle=0 .
\end{aligned}
$$

The claim above allows for a considerable simplification of Eqs. (21) and (22). Each of the peaks in the 2D spectrum keeps track of fewer elements of the process matrix $\chi(T)$ upon isotropic averaging: Only population to population and coherence to coherence transfers can be monitored. For clarity, we display the results for the four peaks of an isotropically averaged 2D spectra in Tables I and II. We introduce the notation $\langle\cdot\rangle_{e_{1} e_{2} e_{3} e_{4}}$, which denotes the isotropically averaged signal stemming from the two pulse polarization configurations $\left(\boldsymbol{e}_{1}, \boldsymbol{e}_{2}, \boldsymbol{e}_{3}, \boldsymbol{e}_{4}\right)=(\boldsymbol{z}, \boldsymbol{z}, \boldsymbol{z}, \boldsymbol{z}),(\boldsymbol{z}, \boldsymbol{z}, \boldsymbol{x}, \boldsymbol{x})$, so that the terms $\left\langle S_{m n}(T)\right\rangle_{e_{1} e_{2} e_{3} e_{4}}$ and $\left\langle S\left(\omega_{\tau}, T, \omega_{t}\right)\right\rangle_{e_{1} e_{2} e_{3} e_{4}}$ have the obvious meanings.

We focus our attention on experiments with short pulses that are broadband enough to create either exciton $|\alpha\rangle$ or $|\beta\rangle$ with the same amplitude, that is, $C_{\omega_{i}}^{p}=C$, for a purely

TABLE I. Isotropically averaged 2D-ES peak amplitudes for the $z z z z$ configuration.

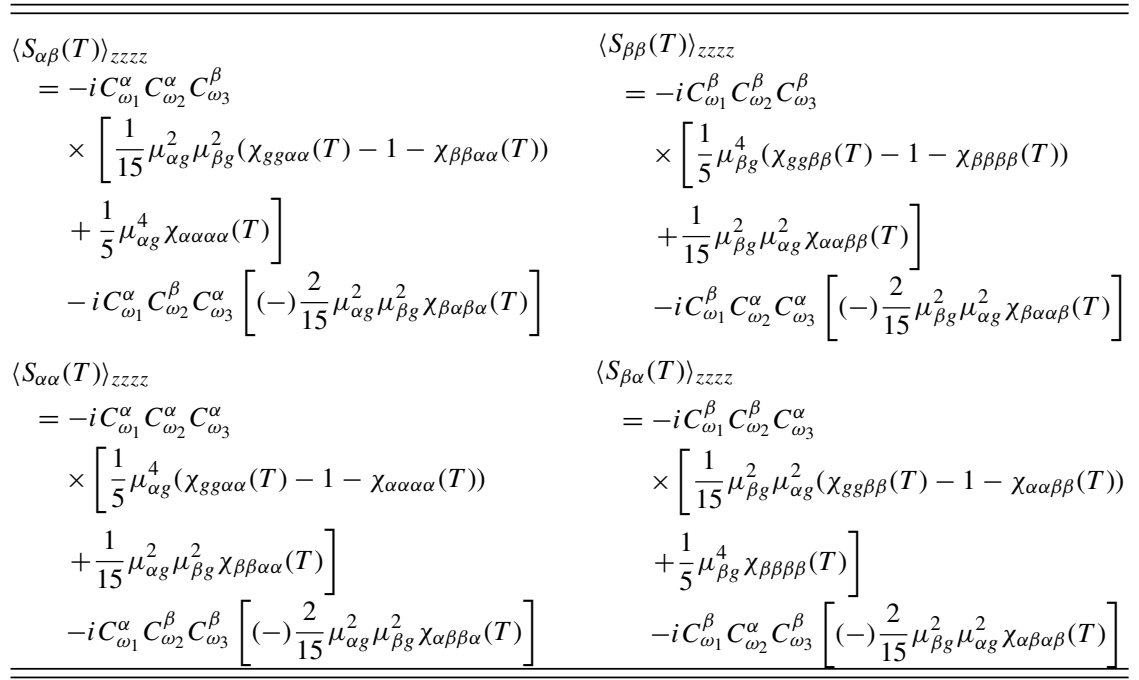


TABLE II. Isotropically averaged 2D-ES peak amplitudes for the $z \boldsymbol{z} \boldsymbol{x} \boldsymbol{x}$ configuration.

\begin{tabular}{|c|c|}
\hline $\begin{array}{l}\left\langle S_{\alpha \beta}(T)\right\rangle_{z z x x} \\
\quad=-i C_{\omega_{1}}^{\alpha} C_{\omega_{2}}^{\alpha} C_{\omega_{3}}^{\beta}\end{array}$ & $\begin{array}{l}\left\langle S_{\beta \beta}(T)\right\rangle_{z z x x} \\
\quad=-i C_{\omega_{1}}^{\beta} C_{\omega_{2}}^{\beta} C_{\omega_{3}}^{\beta}\end{array}$ \\
\hline$\times\left[\frac{2}{15} \mu_{\alpha g}^{2} \mu_{\beta g}^{2}\left(\chi_{g g \alpha \alpha}(T)-1-\chi_{\beta \beta \alpha \alpha}(T)\right)\right.$ & $\times\left[\frac{1}{15} \mu_{\beta g}^{4}\left(\chi_{g g \beta \beta}(T)-1-\chi_{\beta \beta \beta \beta}(T)\right)\right.$ \\
\hline$\left.+\frac{1}{15} \mu_{\alpha g}^{4} \chi_{\alpha \alpha \alpha \alpha}(T)\right]$ & $\left.+\frac{2}{15} \mu_{\beta g}^{2} \mu_{\alpha g}^{2} \chi_{\alpha \alpha \beta \beta}(T)\right]$ \\
\hline$-i C_{\omega_{1}}^{\alpha} C_{\omega_{2}}^{\beta} C_{\omega_{3}}^{\alpha}\left[(+) \frac{1}{15} \mu_{\alpha g}^{2} \mu_{\beta g}^{2} \chi_{\beta \alpha \beta \alpha}(T)\right]$ & $-i C_{\omega_{1}}^{\beta} C_{\omega_{2}}^{\alpha} C_{\omega_{3}}^{\alpha}\left[(+) \frac{1}{15} \mu_{\beta g}^{2} \mu_{\alpha g}^{2} \chi_{\beta \alpha \alpha \beta}(T)\right]$ \\
\hline$\left\langle S_{\alpha \alpha}(T)\right\rangle_{z z x x}$ & $\left\langle S_{\beta \alpha}(T)\right\rangle_{z z x x}$ \\
\hline$=-i C_{\omega_{1}}^{\alpha} C_{\omega_{2}}^{\alpha} C_{\omega_{3}}^{\alpha}$ & $=-i C_{\omega_{1}}^{\beta} C_{\omega_{2}}^{\beta} C_{\omega_{3}}^{\alpha}$ \\
\hline$\times\left[\frac{1}{15} \mu_{\alpha g}^{4}\left(\chi_{g g \alpha \alpha}(T)-1-\chi_{\alpha \alpha \alpha \alpha}(T)\right)\right.$ & $\times\left[\frac{2}{15} \mu_{\beta g}^{2} \mu_{\alpha g}^{2}\left(\chi_{g g \beta \beta}(T)-1-\chi_{\alpha \alpha \beta \beta}(T)\right)\right.$ \\
\hline$\left.+\frac{2}{15} \mu_{\alpha g}^{2} \mu_{\beta g}^{2} \chi_{\beta \beta \alpha \alpha}(T)\right]$ & $\left.+\frac{1}{15} \mu_{\beta g}^{4} \chi_{\beta \beta \beta \beta}(T)\right]$ \\
\hline$-i C_{\omega_{1}}^{\alpha} C_{\omega_{2}}^{\beta} C_{\omega_{3}}^{\beta}\left[(+) \frac{1}{15} \mu_{\alpha g}^{2} \mu_{\beta g}^{2} \chi_{\alpha \beta \beta \alpha}(T)\right]$ & $-i C_{\omega_{1}}^{\beta} C_{\omega_{2}}^{\alpha} C_{\omega_{3}}^{\beta}\left[(+) \frac{1}{15} \mu_{\beta g}^{2} \mu_{\alpha g}^{2} \chi_{\alpha \beta \alpha \beta}(T)\right]$ \\
\hline
\end{tabular}

imaginary constant $C$, for all $p$ and $\omega_{i}$. This condition can be easily relaxed, but we shall proceed with it to analyze our QPT protocol with more detail. Using the condition of Eq. (3), we can eliminate the variables $\chi_{g g \alpha \alpha}(T)$ and $\chi_{g g \beta \beta}(T)$ for $\chi_{\alpha \alpha \alpha \alpha}(T), \chi_{\beta \beta \alpha \alpha}(T), \chi_{\alpha \alpha \beta \beta}(T), \chi_{\beta \beta \beta \beta}(T)$. Also, taking ad- vantage of Eq. (4), we discard $\chi_{\beta \alpha \beta \alpha}(T)$ and $\chi_{\alpha \beta \beta \alpha}(T)$ and keep $\chi_{\alpha \beta \alpha \beta}(T)$ and $\chi_{\beta \alpha \alpha \beta}(T)$. From the left column (LC) of the real and imaginary parts of the spectra (see left columns of Tables I and II), we derive the following real valued matrix equation:

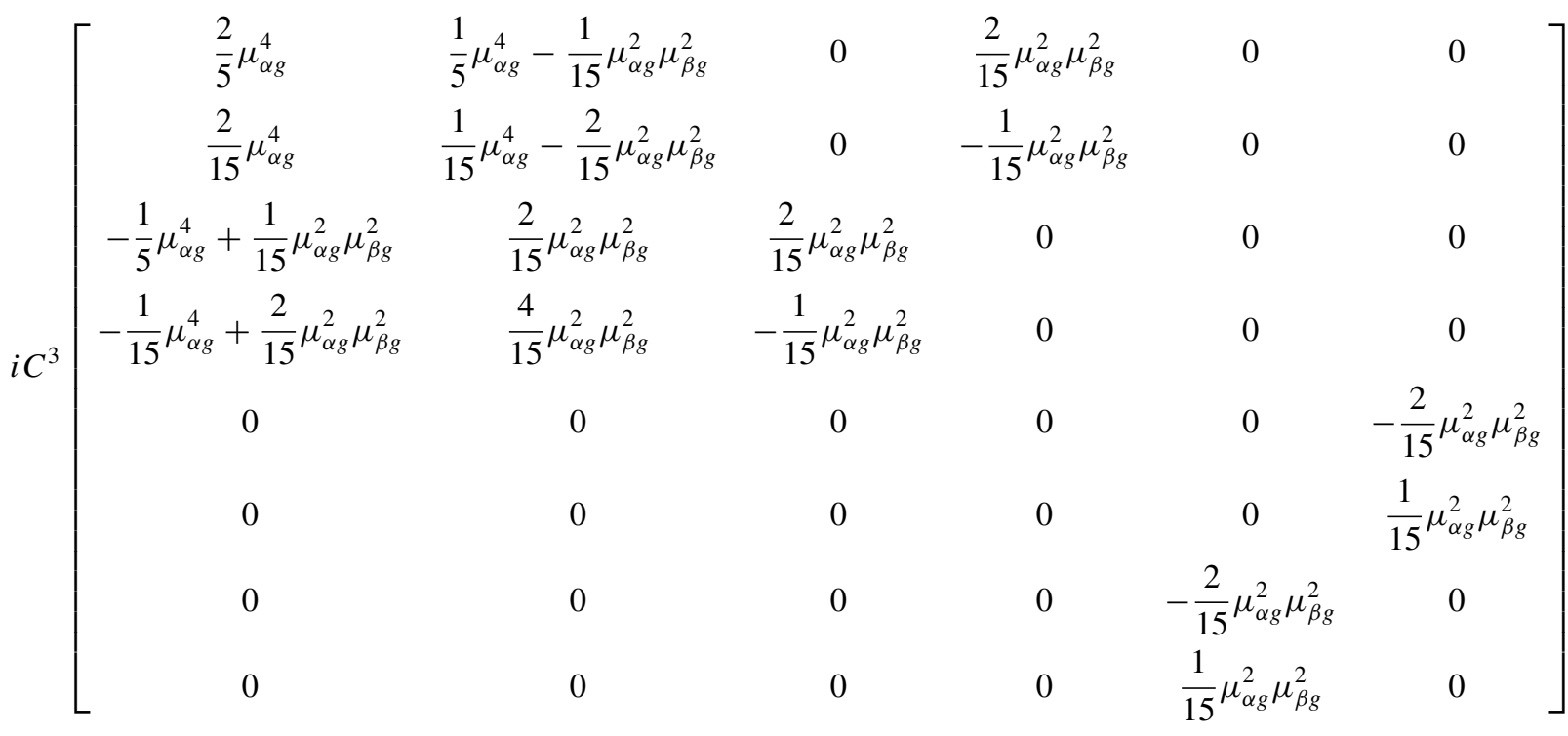

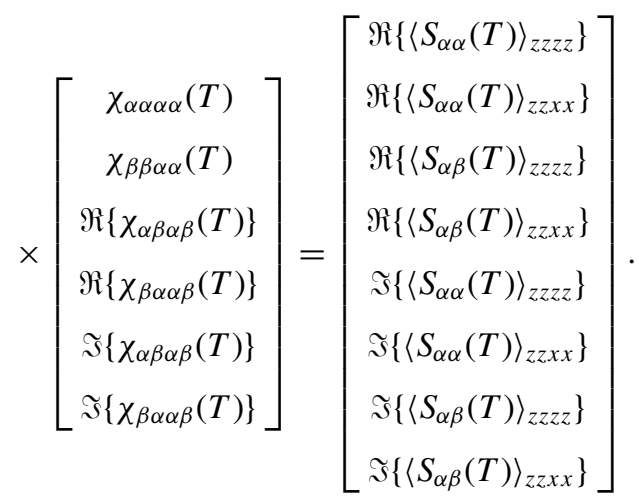


Similarly, the right column (RC) of the spectra yields:

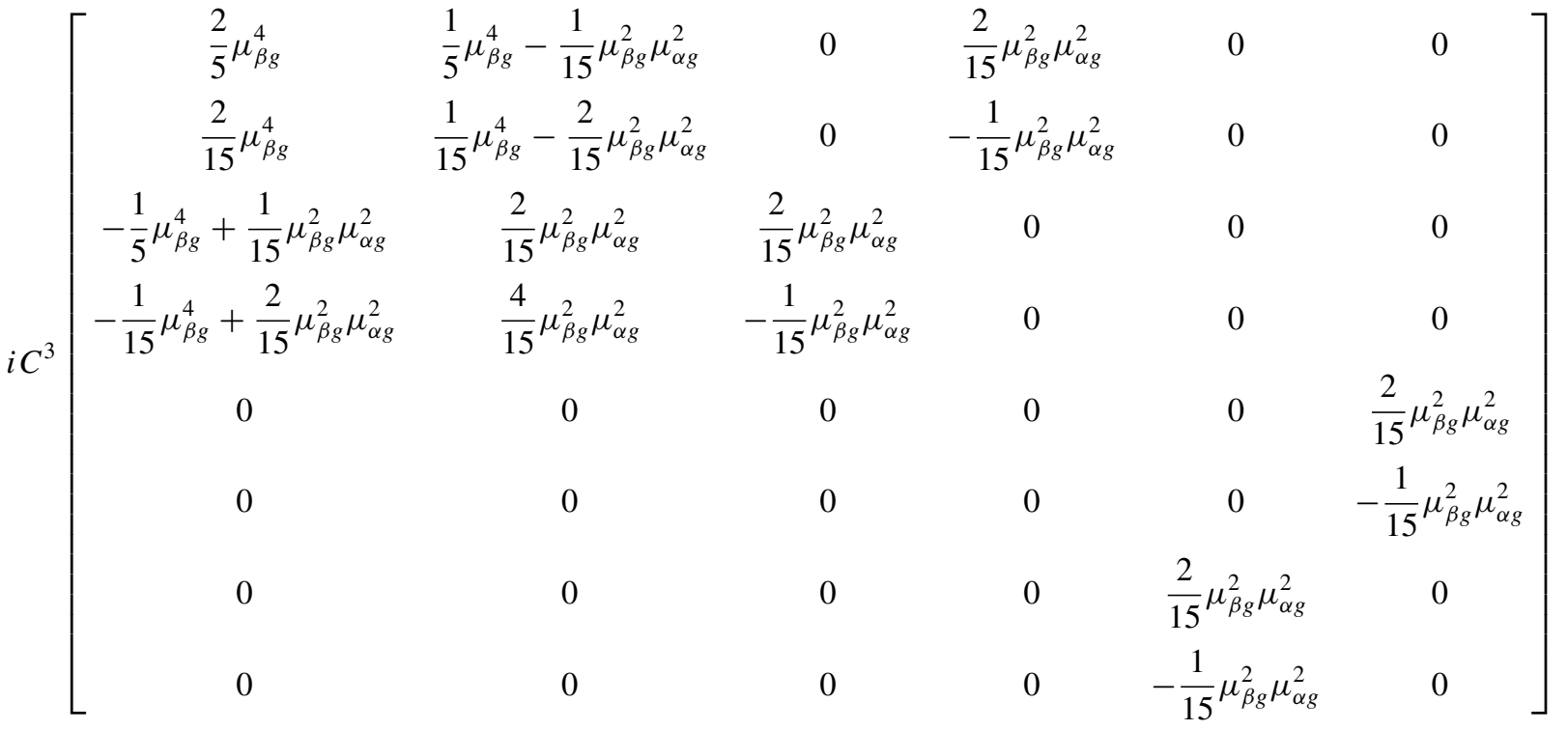

$$
\begin{aligned}
& \times\left[\begin{array}{c}
\chi_{\beta \beta \beta \beta}(T) \\
\chi_{\alpha \alpha \beta \beta}(T) \\
\Re\left\{\chi_{\alpha \beta \alpha \beta}(T)\right\} \\
\Re\left\{\chi_{\beta \alpha \alpha \beta}(T)\right\} \\
\Im\left\{\chi_{\alpha \beta \alpha \beta}(T)\right\} \\
\Im\left\{\chi_{\beta \alpha \alpha \beta}(T)\right\}
\end{array}\right]=\left[\begin{array}{c}
\Re\left\{\left\langle S_{\beta \beta}(T)\right\rangle_{z z z z}\right\} \\
\Re\left\{\left\langle S_{\beta \beta}(T)\right\rangle_{z z x x}\right\} \\
\Re\left\{\left\langle S_{\beta \alpha}(T)\right\rangle_{z z z z}\right\} \\
\Re\left\{\left\langle S_{\beta \alpha}(T)\right\rangle_{z z x x}\right\} \\
\Im\left\{\left\langle S_{\beta \beta}(T)\right\rangle_{z z z z}\right\} \\
\Im\left\{\left\langle S_{\beta \beta}(T)\right\rangle_{z z x x}\right\} \\
\Im\left\{\left\langle S_{\beta \alpha}(T)\right\rangle_{z z z z}\right\} \\
\Im\left\{\left\langle S_{\beta \alpha}(T)\right\rangle_{z z x x}\right\}
\end{array}\right] .
\end{aligned}
$$

Inverting Eqs. (31) and (32) yields most of the elements of $\chi(T)$ involving the single-exciton manifold. While the presented QPT for the homodimer is partial, no complicated pulse shaping efforts need to be carried out. Instead, the requirement is standard pulse polarization control achievable with current experimental capabilities. ${ }^{11,12,72-77}$

The transition dipole moments must be well characterized in order to construct the matrices in Eqs. (31) and (32). This requirement is self-consistently fulfilled by only collecting the spectra in the collinear and cross-polarized configurations. Notice that $\chi_{\alpha \alpha \alpha \alpha}(T)$ and $\chi_{\beta \beta \alpha \alpha}(T)$ are exclusively monitored in the $\mathrm{LC}$, and $\chi_{\beta \beta \beta \beta}(T)$ and $\chi_{\alpha \alpha \beta \beta}(T)$ only detected in the RC. However, coherence transfer terms $\chi_{\alpha \beta \alpha \beta}(T)$ and $\chi_{\beta \alpha \alpha \beta}(T)$ are repeatedly monitored in different peaks in both sides of the spectra. Due to this repetition, there are redundant equations that allow for the self-consistent extraction of the angle $\phi$ without compromising the inversion of the elements of $\chi(T)$. Details about this parameter extraction are developed in subsection E. For the time being, we assume that the information about the transition dipoles is previously known.

\section{Stability of the quantum process tomography protocol for a homodimer}

In order to characterize the stability of inversion of $\chi(T)$, we can arrange Eqs. (31) and (32) into a single matrix equation $\boldsymbol{M} \vec{\chi}(T)=\vec{S}(T)$, where $\boldsymbol{M}$ is a $16 \times 8$ matrix of dipole moments, $\vec{\chi}(T)$ is a vector of 8 unknowns, and $\vec{S}(T)$ is a vector of 16 real valued amplitudes extracted from the signal. Denoting with $\|\cdot\|$ the spectral norm of a vector or a matrix, ${ }^{78}$ we obtain a bound on the relative error of the inverted vector $\vec{\chi}(T)$ which yields the QPT:

$$
\frac{\|\Delta \vec{\chi}(T)\|}{\|\vec{\chi}(T)\|} \leq \kappa \frac{\|\Delta S(T)\|}{\|S(T)\|}
$$

where $\Delta \vec{\chi}(T)$ and $\Delta S(T)$ denote errors in $\vec{\chi}(T)$ and $S(T)$ upon inversion, respectively, and the condition number $\kappa$ is given by

$$
\kappa=\|M\|\left\|M^{-1}\right\| .
$$

The lowest possible value for a condition number is $\kappa=1$. In Fig. (6), a plot of $\kappa$ versus $\phi$ (red) is displayed. As expected, very large values of $\kappa$, which denote unstable in- 
versions, are expected for systems where the site dipoles are aligned or antialigned, when one of the eigenstates of $H_{S}$ becomes dark. We also indicate a range of angles where $\kappa$ is below an acceptable threshold, say $\kappa \leq 200$ (blue horizontal line), which consists of angles in the range $0.12 \pi \leq \phi$ $\leq 0.88 \pi$. Also, note that $\kappa$ is symmetric about the minimum of $\kappa$ at $\phi=\pi / 2$, where the best inversion is carried out with $\kappa \approx 3.9$.

\section{Analytical expressions for $\chi(T)$}

To gain insights into the QPT protocol, we derive explicit expressions for the elements of $\chi(T)$ in terms of the amplitudes of the spectra and the angle $\phi$ between the dipoles. First, we substitute Eqs. (29) and (30) into the expression associated with the LC of the spectra in Eq. (31). The following relations are obtained after inverting the resulting matrix equation:

$$
\begin{aligned}
\chi_{g g \alpha \alpha}(T)-1= & -\frac{3 \sec ^{2}(\phi / 2)}{\left(i C^{3}\right)\left(4 d^{4}\right)} \\
& \times \Re\left\{\left\langle S_{\alpha \alpha}(T)\right\rangle_{z z z z}+2\left\langle S_{\alpha \alpha}(T)\right\rangle_{z z x x}\right. \\
& \left.+\left\langle S_{\alpha \beta}(T)\right\rangle_{z z z z}+2\left\langle S_{\alpha \beta}(T)\right\rangle_{z z x x}\right\},
\end{aligned}
$$

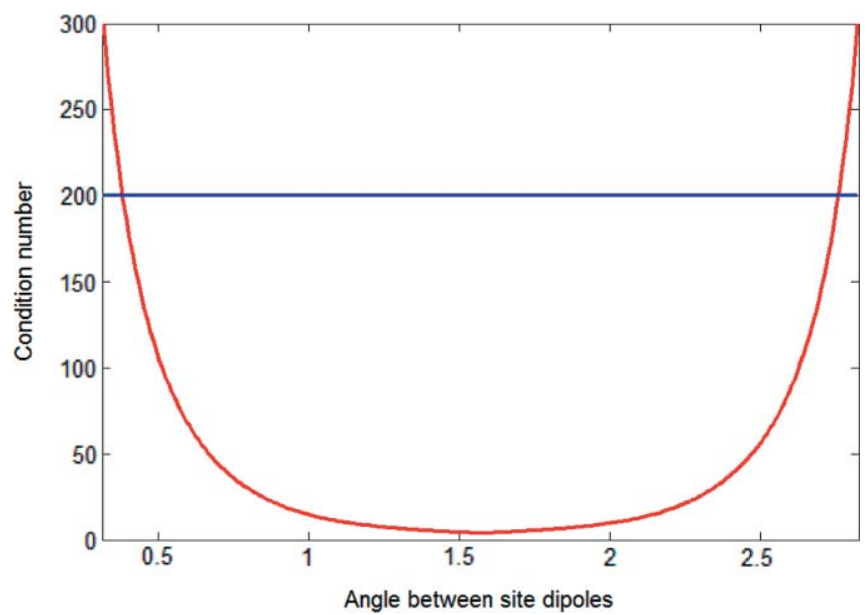

FIG. 6. Stability of the homodimer QPT protocol. As described in the text, the QPT protocol depends on the inversion of a matrix which is a function of transition dipole moments. The condition number of the matrix $(\kappa)$ vs the angle between site dipoles $(\phi)$ is plotted in red. The blue line plots the constant value of $\kappa=200$, as a reference to indicate that for the range of angles $0.12 \pi \leq \phi \leq 0.88 \pi$, the condition number is below that value.

$$
\begin{aligned}
\Re\left\{\chi_{\alpha \beta \alpha \beta}(T)\right\}= & \frac{3}{\left(i C^{3}\right)\left(4 d^{4}\right)} \\
& \times \Re\left\{2\left(\left\langle S_{\alpha \alpha}(T)\right\rangle_{z z z z}+2\left\langle S_{\alpha \alpha}(T)\right\rangle_{z z x x}\right)\right. \\
& +\left(\cot ^{2} \frac{\phi}{2}+2 \tan ^{2} \frac{\phi}{2}+5\right)\left\langle S_{\alpha \beta}(T)\right\rangle_{z z z z} \\
& \left.-\left(3 \cot ^{2} \frac{\phi}{2}+\tan ^{2} \frac{\phi}{2}\right)\left\langle S_{\alpha \beta}(T)\right\rangle_{z z x x}\right\}
\end{aligned}
$$

$$
\begin{aligned}
\Re\left\{\chi_{\beta \alpha \alpha \beta}(T)\right\}= & \frac{3}{\left(i C^{3}\right)\left(4 d^{4}\right)} \\
& \times \Re\left\{\left(\cot ^{2} \frac{\phi}{2}+2 \tan ^{2} \frac{\phi}{2}+1\right)\left\langle S_{\alpha \alpha}(T)\right\rangle_{z z z z}\right. \\
& -\left(3 \cot ^{2} \frac{\phi}{2}+\tan ^{2} \frac{\phi}{2}+8\right)\left\langle S_{\alpha \alpha}(T)\right\rangle_{z z x x} \\
& \left.-2\left(\left\langle S_{\alpha \beta}(T)\right\rangle_{z z z z}+2\left\langle S_{\alpha \beta}(T)\right\rangle_{z z x x}\right)\right\}, \quad(39) \\
\Im\left\{\chi_{\alpha \beta \alpha \beta}(T)\right\}= & \frac{15}{\left(i C^{3}\right)\left(8 d^{4}\right)} \Im\left\{\left(\csc ^{2} \frac{\phi}{2} \sec ^{2} \frac{\phi}{2}\right)\left\langle S_{\alpha \beta}(T)\right\rangle_{z z z z}\right\} \\
= & \frac{15}{\left(i C^{3}\right)\left(4 d^{4}\right)} \mathfrak{s}\left\{\left(\csc ^{2} \frac{\phi}{2} \sec ^{2} \frac{\phi}{2}\right)\left\langle S_{\alpha \beta}(T)\right\rangle_{z z x x}\right\}, \\
\Im\left\{\chi_{\beta \alpha \alpha \beta}(T)\right\}= & -\frac{15}{\left(i C^{3}\right)\left(8 d^{4}\right)} \mathfrak{\Im}\left\{\left(\csc ^{2} \frac{\phi}{2} \sec ^{2} \frac{\phi}{2}\right)\left\langle S_{\alpha \alpha}(T)\right\rangle_{z z z z}\right\} \\
= & \frac{15}{\left(i C^{3}\right)\left(4 d^{4}\right)} \Im\left\{\left(\csc ^{2} \frac{\phi}{2} \sec ^{2} \frac{\phi}{2}\right)\left\langle S_{\alpha \alpha}(T)\right\rangle_{z z x x}\right\} .
\end{aligned}
$$

Similarly, the RC of the spectra through Eq. (32) yields expressions for $\chi_{g g \beta \beta}(T)-1, \chi_{\beta \beta \beta \beta}(T), \chi_{\alpha \alpha \beta \beta}(T)$, and again, the real and imaginary parts of $\chi_{\alpha \beta \alpha \beta}(T)$ and $\chi_{\beta \alpha \alpha \beta}(T)$. These expressions follow from the ones right above by the substitutions $\{\alpha, \beta\} \rightarrow\{\beta, \alpha\}$ and $\phi \rightarrow \pi-\phi$ [see Eqs. (30)].

From Eq. (40) of the LC as well as the analogues of the RC of the spectra, we notice that the imaginary parts of $\left\langle S_{\alpha \beta}(T)\right\rangle_{z z z z},\left\langle S_{\alpha \beta}(T)\right\rangle_{z z x x},\left\langle S_{\beta \alpha}(T)\right\rangle_{z z x x}$, and $\left\langle S_{\beta \alpha}(T)\right\rangle_{z z x x}$, are all exclusively proportional to $\mathfrak{s}\left\{\chi_{\alpha \beta \alpha \beta}(T)\right\}$. The least-squares solution for $\mathfrak{\Im}\left\{\chi_{\alpha \beta \alpha \beta}(T)\right\}$ using Eqs. (31) and (32) is just the average of these four values; the analogous conclusion holds for $\Im\left\{\chi_{\beta \alpha \alpha \beta}(T)\right\}$. The real parts, $\mathfrak{i}\left\{\chi_{\alpha \beta \alpha \beta}(T)\right\}, \mathfrak{R}\left\{\chi_{\beta \alpha \alpha \beta}(T)\right\}$ satisfy less trivial relationships. Each of them appears twice in the equations, once in the $\mathrm{RC}$ and another time in the LC of the spectra. It is this redundancy in the spectral information what allows for the extraction of the angle between the dipoles.

\section{E. Determination of the angle $\varphi$ between the two dipoles}

Equating the values of $\mathfrak{R}\left\{\chi_{\alpha \beta \alpha \beta}(T)\right\}$ from the expressions in Eq. (38) and its RC analogue results in the following 
quadratic equation in $\xi=\tan ^{2}\left(\frac{\phi}{2}\right)$ :

$$
\begin{aligned}
& \Re\left\{\left[\left\langle S_{\beta \alpha}(T)\right\rangle_{z z z z}-3\left\langle S_{\beta \alpha}(T)\right\rangle_{z z x x}-2\left\langle S_{\alpha \beta}(T)\right\rangle_{z z z z}\right.\right. \\
& \left.+\left\langle S_{\alpha \beta}(T)\right\rangle_{z z x x}\right] \xi^{2} \\
& +\left[5\left\langle S_{\beta \alpha}(T)\right\rangle_{z z z z}+2\left\langle S_{\beta \beta}(T)\right\rangle_{z z z z}+4\left\langle S_{\beta \beta}(T)\right\rangle_{z z x x}\right. \\
& \left.-5\left\langle S_{\alpha \beta}(T)\right\rangle_{z z z z}-2\left\langle S_{\alpha \alpha}(T)\right\rangle_{z z z z}-4\left\langle S_{\alpha \alpha}(T)\right\rangle_{z z x x}\right] \xi \\
& +\left[2\left\langle S_{\beta \alpha}(T)\right\rangle_{z z z z}-\left\langle S_{\beta \alpha}(T)\right\rangle_{z z x x}-\left\langle S_{\alpha \beta}(T)\right\rangle_{z z z z}\right. \\
& \left.\left.+3\left\langle S_{\alpha \beta}(T)\right\rangle_{z z x x}\right]\right\}=0 .
\end{aligned}
$$

A similar expression can be found for $\Re\left\{\chi_{\beta \alpha \alpha \beta}(T)\right\}$ from Eq. (39) and its RC analogue:

$$
\begin{aligned}
& \Re\left\{\left[2\left\langle S_{\alpha \alpha}(T)\right\rangle_{z z z z}-\left\langle S_{\alpha \alpha}(T)\right\rangle_{z z x x}-\left\langle S_{\beta \beta}(T)\right\rangle_{z z z z}\right.\right. \\
& \left.+3\left\langle S_{\beta \beta}(T)\right\rangle_{z z x x}\right] \xi^{2} \\
& +\left[\left\langle S_{\alpha \alpha}(T)\right\rangle_{z z z z}-8\left\langle S_{\alpha \alpha}(T)\right\rangle_{z z x x}-2\left\langle S_{\alpha \beta}(T)\right\rangle_{z z z z}\right. \\
& -4\left\langle S_{\alpha \beta}(T)\right\rangle_{z z x x} \\
& -\left\langle S_{\beta \beta}(T)\right\rangle_{z z z z}+8\left\langle S_{\beta \beta}(T)\right\rangle_{z z x x}+2\left\langle S_{\beta \alpha}(T)\right\rangle_{z z z z} \\
& \left.+4\left\langle S_{\beta \alpha}(T)\right\rangle_{z z x x}\right] \xi \\
& +\left[\left\langle S_{\alpha \alpha}(T)\right\rangle_{z z z z}-3\left\langle S_{\alpha \alpha}(T)\right\rangle_{z z x x}-2\left\langle S_{\beta \beta}(T)\right\rangle_{z z z z}\right. \\
& \left.\left.+\left\langle S_{\beta \beta}(T)\right\rangle_{z z x x}\right]\right\}=0 .
\end{aligned}
$$

The identities in Eqs. (42) and (43) are remarkable in the sense that they are satisfied at every waiting time $T$ : they do not depend on short time coherent dynamics or long time asymptotic behavior. By monitoring the peak amplitudes of the spectra arising from the two different polarization configurations, the angle $\phi$ between the two site dipoles can be readily extracted using either expression. This determination is robust because it can be repeated for every value of $T$ for which the signal has been collected.

\section{F. Summary of quantum process tomography protocol for a coupled homodimer}

We proceed to summarize the algorithm of the QPT protocol for a coupled homodimer.

(1) Obtain the amplitudes $\left\langle S_{m n}(T)\right\rangle_{e_{1} e_{2} e_{3} e_{4}}$ for $m, n \in$ $\{\alpha, \beta\}$ and the two polarization settings $\left(\boldsymbol{e}_{1}, \boldsymbol{e}_{2}, \boldsymbol{e}_{3}, \boldsymbol{e}_{4}\right)$ $=(\boldsymbol{z}, \boldsymbol{z}, \boldsymbol{z}, \boldsymbol{z}),(\boldsymbol{x}, \boldsymbol{x}, \boldsymbol{z}, \boldsymbol{z})$. This information can be extracted from the two respective polarization controlled 2D-ES, $\left\langle S\left(\omega_{\tau}, T, \omega_{t}\right)\right\rangle_{e_{1} e_{2} e_{3} e_{4}}$. For simplicity, all the pulses are taken to be of the same duration (short compared to the time scales of excited state dynamics).

(2) Extract the angle $\phi$ from the data of step 1 and Eqs. (42) and (43).

(3) Plug in information obtained from step 1 and the angle $\phi$ from step 2 into the expressions for the elements of $\chi(T)$ in Eqs. (35)-(41) and their RC analogues. Some important observations: (a) These expressions are all proportional to the factor $\left(C^{3} d^{4}\right)^{-1}$. The norm of the dipole $d$ can be extracted from the intensity of the absorption spectrum of the monomer. Alternatively, we can assume that $\chi_{g g \alpha \alpha}(T), \chi_{g g \beta \beta} \approx 0$ during femtosecond time scales, so that Eq. (35) or its RC analogue can be used to extract the constant factors. Finally, if this information is not readily available, the results are known up to this constant factor. (b) By construction from Eq. (42), the calculated value of $\Re\left\{\chi_{\alpha \beta \alpha \beta}(T)\right\}$ will be the same using either Eq. (38) or its RC analogue. The same holds for Eqs. (43), $\Re\left\{\chi_{\beta \alpha \alpha \beta}(T)\right\}$, and (38) and its respective $\mathrm{RC}$ analogue.

\section{G. Numerical example}

In this subsection, we illustrate the described QPT protocol with a model homodimer. Marcus and co-workers have recently reported a synthetic system of porphyrin molecules, which self-assemble into homodimers in the presence of liposomes. ${ }^{79}$ The parameters of this system, extracted from phase-modulation electronic coherence spectroscopy, are $\bar{\omega}=16633 \mathrm{~cm}^{-1}, J=175 \mathrm{~cm}^{-1}$, and $\phi=65^{\circ}$. Information on the spectral density of this system is not available in the literature yet. We adopt a simple system-bath model based on the secular Redfield approach and independent bath approximation (IBA) for each site. The weak system-bath model is reasonable since porphyrins are rigid molecules which change their structures minimally upon electronic excitation. The IBA must be re-examined, since the liposome media guarantee a bath that could be strongly correlated in both sites. Nevertheless, the purpose of this example is not to provide an exact account of the excited state dynamics of this system but rather an illustration of the QPT protocol using reasonable time scales that one might encounter in a realistic setting. Furthermore, a careful study of the precise bath-induced dynamics would probably include non-Markovian effects. ${ }^{35,80-82}$ All of these contributions effects are beyond the scope of this study and shall be addressed in future work in collaboration with an experimental realization. However, we remind the reader that the proposed QPT is valid for both Markovian and non-Markovian baths.

We consider a harmonic bath with an Ohmic spectral density: $J(\omega)=\lambda / \omega_{c} \omega e^{-\omega / \omega_{c}}$, with $\lambda=100 \mathrm{~cm}^{-1}$, $\omega_{c}=150 \mathrm{~cm}^{-1}$ at a temperature $\mathcal{T}=273 \mathrm{~K}$. Identical baths are assumed to be linearly coupled to each of the sites. We closely follow the calculation reported in Ref. 83 and adapted in Ref. 42. The dynamics of the total excitonic system, which is a proper density matrix, is governed by the following equation of motion:

$$
\dot{\rho}(T)=-i\left[H_{S}, \rho(T)\right]-\mathcal{R} \rho(T),
$$

where $\mathcal{R}$ denotes the time-independent sparse dissipative superoperator containing only a few nonzero elements listed in Table III. Since $\dot{\rho}(T)$ only depends on $\rho(T)$ and not on the value of the quantum state at previous times, the simulated dynamics are Markovian.

It is well known that the secular Redfield equations guarantee thermal equilibrium since the population transfer rates satisfy $R_{\alpha \alpha \beta \beta} / R_{\beta \beta \alpha \alpha}=e^{-\omega_{\alpha \beta} / k_{B} \mathcal{T}}$, where $k_{B}$ is the Boltzmann constant. Also, $R_{f g f g}$ will not be relevant for the calculations, as coherences between the ground state and the biexciton are never created in the PE experiment. The free-induction decay 
TABLE III. Values (in $f s^{-1}$ ) of nonzero rates of the secular Redfield tensor.

\begin{tabular}{cc}
\hline \hline$R_{\beta \beta \alpha \alpha}$ & $8.02 \times 10^{-4}$ \\
$R_{\alpha \alpha \beta \beta}=e^{-\omega_{\alpha \beta} / k_{B} T} R_{\beta \beta \alpha \alpha}$ & $5.07 \times 10^{-3}$ \\
$R_{\alpha \beta \alpha \beta}=R_{\beta \alpha \beta \alpha}$ & $2.93 \times 10^{-3}$ \\
$R_{\alpha g \alpha g}=R_{g \alpha g \alpha}=R_{f \alpha f \alpha}=R_{\alpha f \alpha f}$ & $1.23 \times 10^{-2}$ \\
$R_{\beta g \beta g}=R_{g \beta g \beta}=R_{f \beta f \beta}=R_{\beta f \beta f}$ & $1.45 \times 10^{-2}$ \\
$R_{f g f g}=R_{g f g f}$ & $4.77 \times 10^{-2}$ \\
\hline
\end{tabular}

rates for the coherence and echo intervals will be taken for simplicity to be the same, $\Gamma_{\alpha g} \approx \Gamma_{\beta g} \approx \frac{1}{2}\left(R_{\alpha g \alpha g}+R_{\beta g \beta g}\right)$. This restriction is by no means necessary but will simplify the simulations below.

The nonzero elements of $\chi(T)$ for the single-exciton manifold are presented in Table IV.

In this particular calculation, coupling to the photon bath has been ignored beyond the ultrashort pulses, as spontaneous emission occurs in the order of nanoseconds, i.e., $\chi_{g g \alpha \alpha}(T)$ $=\chi_{g g \beta \beta}(T)=0$. Therefore, Eq. (3) can be readily confirmed as $\chi_{\alpha \alpha \alpha \alpha}(T)+\chi_{\beta \beta \alpha \alpha}(T)=\chi_{\alpha \alpha \beta \beta}(T)+\chi_{\beta \beta \alpha \alpha}(T)=1$. Also, due to the secular approximation, $\chi_{\beta \alpha \alpha \beta}(T)=\left(\chi_{\alpha \beta \beta \alpha}(T)\right)^{*}$ $=0$. The picture of the secular Redfield equations is very simple and provides transparent means for understanding the QPT protocol for the homodimer: The evolution of populations and the coherences independently satisfy standard firstorder kinetic equations, leading to exponential integrated dynamics.

In Fig. 7, we display the calculated 2D-ES of this model system. We consider the three pulses to be identical, centered about $\omega_{1}=\omega_{2}=\omega_{3}=16633 \mathrm{~cm}^{-1}$, of $F W H M=20 \mathrm{fs}$, i.e., $\sigma=8.49 \mathrm{fs}$, which amount to an equal excitation amplitude $C$ for both $|\alpha\rangle$ and $|\beta\rangle$. Rabi oscillations for a coherent superposition between $|\alpha\rangle$ and $|\beta\rangle$ occur with a period $T_{c}=47.5 \mathrm{fs}$. We present several snapshots of the real and imaginary parts of the spectra at values of waiting time $T$ corresponding to multiples of $T_{c} / 2$, skipping $T_{c}=0$, as our theory has avoided pulse overlap effects. In principle, as Eq. (22) and its RC analogue indicate, the excitonic quantum beats associated with the term $\chi_{\alpha \beta \alpha \beta}(T)$ can be monitored by looking at either cross peak of the spectra. This feature is subtly manifested in every column of the figure, but more easily perceived in the real part the $z z x x$ spectrum, where the peak at $\left(\omega_{\beta}, \omega_{\alpha}\right)$ changes from red to yellow/green every interval $T_{c} / 2$ before the bosonic bath has washed out significant portion of the coherent dynamics at about $T=5 T_{c}$. Also, incoherent population transfer primarily from $|\beta\rangle$ to $|\alpha\rangle$ (downhill) manifests as a decrease in amplitude of the peak at $\left(\omega_{\beta}, \omega_{\beta}\right)$ due to ESA and an increase in $\left(\omega_{\beta}, \omega_{\alpha}\right)$ due to SE. ${ }^{2}$ This effect is not very evident in most of the spectra but more obviously seen in the real part of $z z x x$ spectrum. The ambiguities arising from the

TABLE IV. Nonzero elements of $\chi(T)$ involving single-exciton states for the secular Redfield model.

\begin{tabular}{cc}
\hline \hline$\chi_{\alpha \alpha \alpha \alpha}(T)$ & $e^{-R_{\beta \beta \alpha \alpha} T}$ \\
$\chi_{\beta \beta \alpha \alpha}(T)$ & $1-e^{-R_{\beta \beta \alpha \alpha} T}$ \\
$\chi_{\beta \beta \beta \beta}(T)$ & $e^{-R_{\alpha \alpha \beta \beta} T}$ \\
$\chi_{\alpha \alpha \beta \beta}(T)$ & $1-e^{-R_{\alpha \alpha \beta \beta} T}$ \\
$\chi_{\alpha \beta \alpha \beta}(T)=\left(\chi_{\beta \alpha \beta \alpha}(T)\right)^{*}$ & $e^{-i \omega_{\alpha \beta} T} e^{-R_{\alpha \beta \alpha \beta} T}$ \\
\hline \hline
\end{tabular}

qualitative glimpse of these 2D-ES are another compelling reason to adopt a QPT approach to filter out information on the quantum state of the probed system from them.

From the simulated spectra $\left\langle S\left(\omega_{\tau}, T, \omega_{t}\right)\right\rangle_{e_{1} e_{2} e_{3} e_{4}}$, the extraction of the terms $\left\langle S_{m n}(T)\right\rangle_{e_{1} e_{2} e_{3} e_{4}}$ is achieved with high fidelity $(>99 \%)$ by a nonlinear optimization routine based on the simplex search method with bound constraints. ${ }^{84}$ The signals are fitted to a sum of four different resonances as in the isotropically averaged version of Eq. (18). ${ }^{124}$ The parameters $\omega_{m g}, \omega_{n g}, \Gamma$, and $\left\langle S_{m n}(T)\right\rangle_{e_{1} e_{2} e_{3} e_{4}}$ are reconstructed from 2D-ES with a grid spacing of $\Delta \omega_{\tau}=\Delta \omega_{t}=1 \mathrm{~cm}^{-1}$ and a grid size of $1050 \mathrm{~cm}^{-1}$ for every axis. We present the results of this calculation in Fig. 8. Notice that the imaginary parts of the diagonal peaks are zero since no terms of the form $\chi_{\beta \alpha \alpha \beta}(T)=\left(\chi_{\alpha \beta \beta \alpha}(T)\right)^{*}$ are considered in the secular Redfield theory, and population transfer terms are purely real.

Equation (42) is solved at every waiting time $T \in\left[T_{c} / 2\right.$, $10 T_{c}$ ] yielding the roots $\xi=1.000,0.4059$ for every $T$, without variance after the fourth decimal digit, indicating its robustness for the inversion of $\phi$. These values of $\xi$ imply $\phi=90^{\circ}$ or $65^{\circ}$. The same exercise with Eq. (43) gives $\xi=$ $-0.4059,0.4059$, and $\phi=65^{\circ}$ or $86.3 i^{\circ}$. Since both identities need to hold simultaneously, the result $\phi=65^{\circ}$ follows unambiguously, as expected.

Finally, the terms $\left\langle S_{m n}(T)\right\rangle_{e_{1} e_{2} e_{3} e_{4}}$ and the angle $\phi$ allow for the evaluation of the elements of $\chi(T)$ which are extractable for the homodimer. Figure 9 shows that this reconstruction coincides with the analytical expressions presented in Table IV. The population decay terms $\chi_{\alpha \alpha \alpha \alpha}(T), \chi_{\beta \beta \beta \beta}(T)$ both start at 1 and reach 0 exponentially, the second faster than the first, since $|\beta\rangle$ is the excitonic state of higher energy. The population transfer terms $\chi_{\alpha \alpha \beta \beta}(T), \chi_{\beta \beta \alpha \alpha}(T)$ are complementary to the former ones, with the transfer from $|\beta\rangle$ to $|\alpha\rangle$ being faster for the same reasons just mentioned. The coherence term decays exponentially, with real and imaginary parts $\pi / 2$ phase shifted one from another. The calculated time scale of this decay (hundreds of femtoseconds) is similar to the one inferred from the experiment reported by Lee and co-workers, where a superposition of excitons in the bacteriopheophytin and bacteriochlorophyll sites in the reaction center of purple bacteria is monitored indirectly through a two-color experiment. ${ }^{85}$

\section{DISCUSSION}

In the present article, we have outlined a general theory for carrying out the QPT for a molecular dimer using the information contained in various frequency and polarization controlled 2D-ES. We started by providing the basic concepts of QPT and operationally defined a QPT as a protocol to extract the process matrix $\chi(T)$, which in principle, completely characterizes a quantum black box (in our case, the box being the single-exciton manifold of the dimer). After reviewing the model Hamiltonian as well as the transition dipole moments of an excitonic dimer, we adapted the QPT theory presented in our previous work, where the nonlinear polarization was analyzed in real time for single values of $\tau$ and $t$ times [see Eqs. (13) and (14)], ${ }^{42}$ to the more standard and visual Fourier transformed signal collected along several values of these 
(a) $T c / 2$
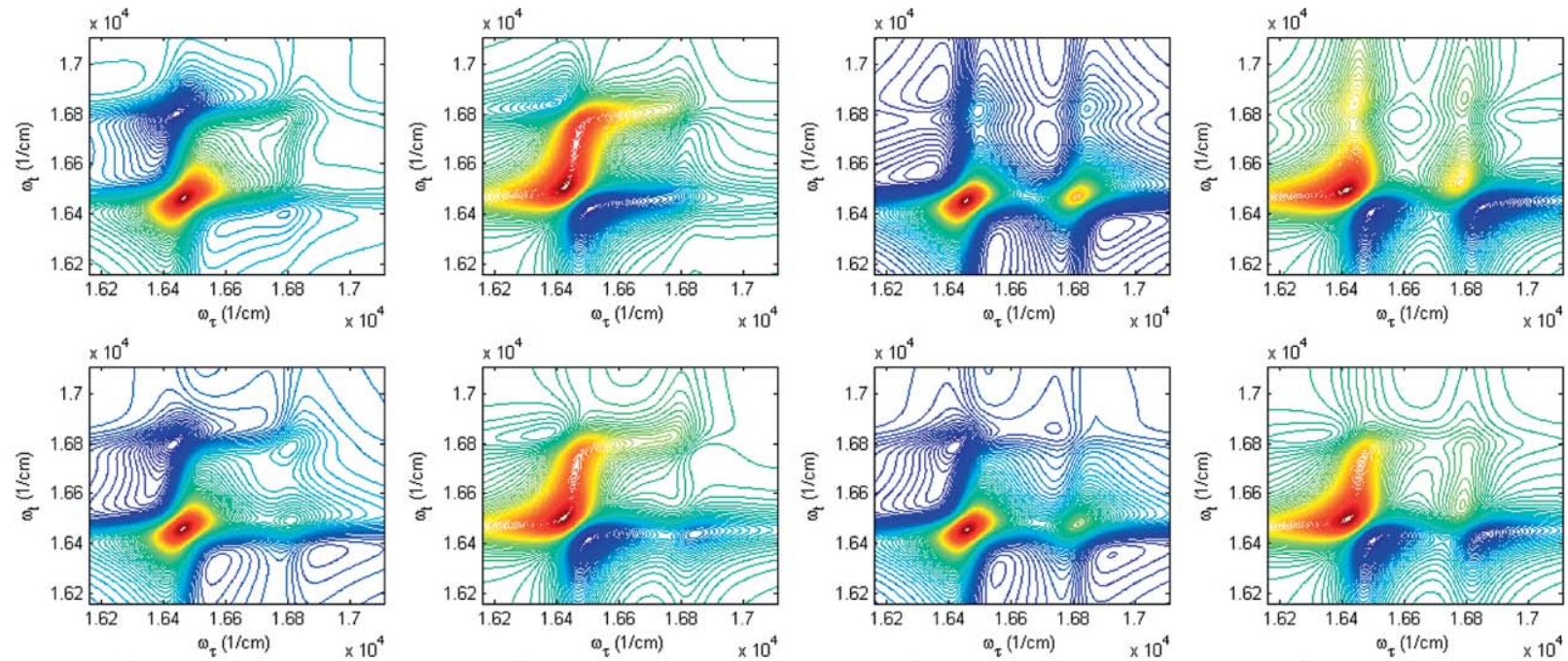

(b) Tc
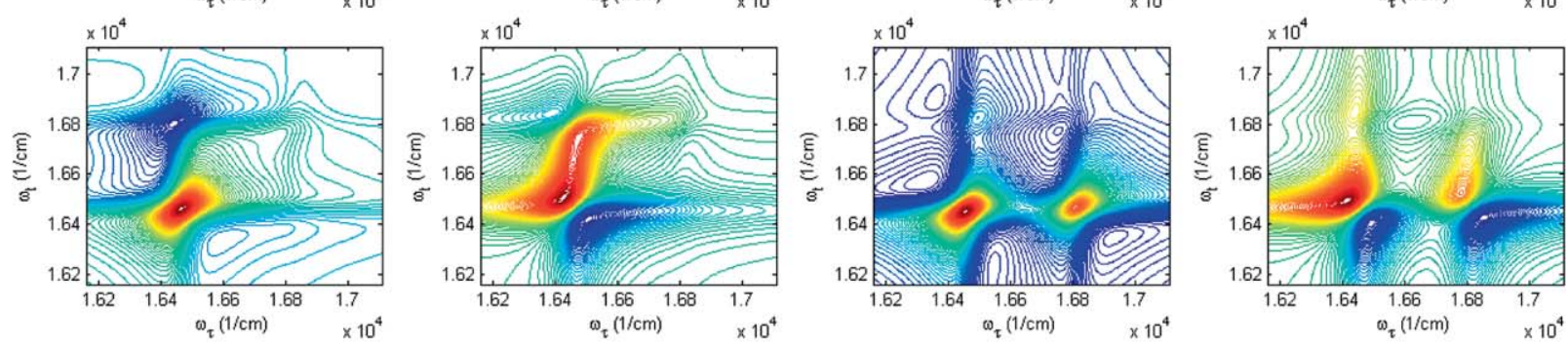

(c) $3 \mathrm{Tc} / 2$
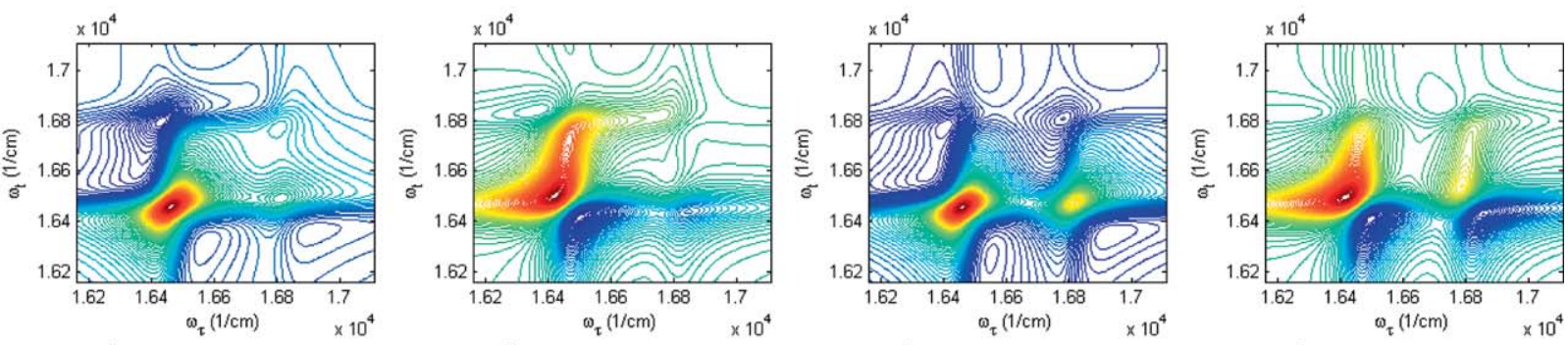

(d) 2Tc
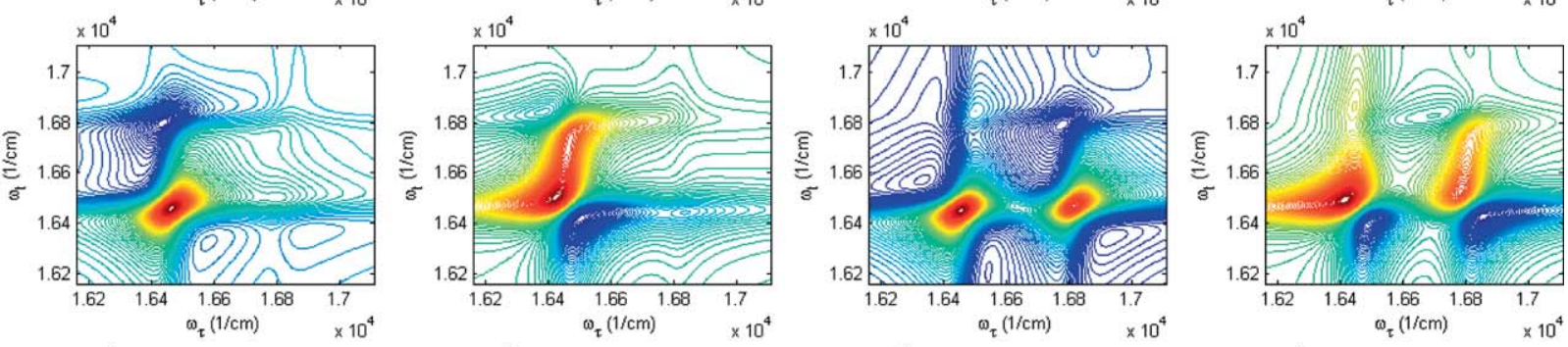

(e) $9 T c / 2$
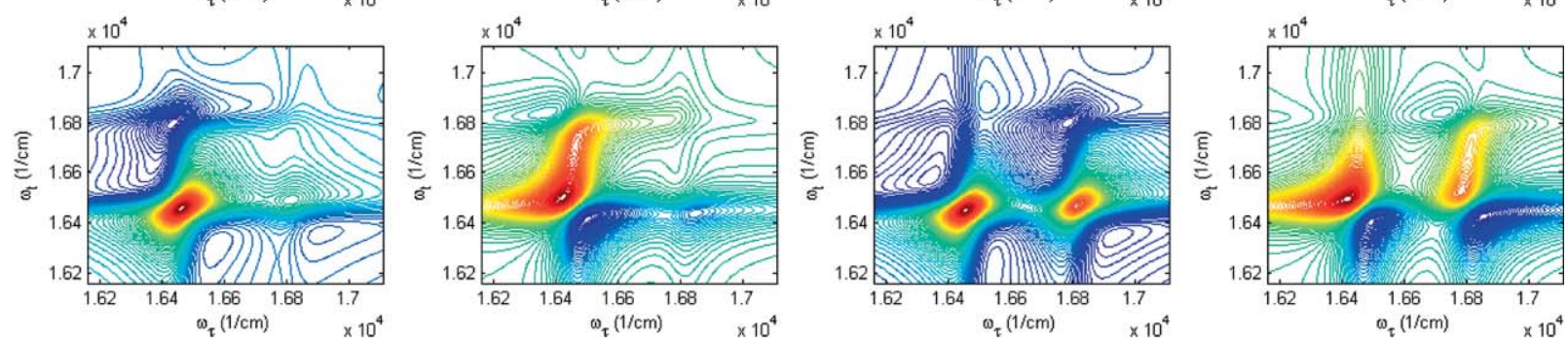

FIG. 7. 2D-ES for coupled porphyrin homodimer with secular Redfield model. From left to right, we show the real and imaginary parts of the spectrum with $z z z z$ polarization setting (first and second columns), and with $z z x x$ setting (third and fourth columns). Each row represents a particular waiting time $T$, corresponding to (a) $T_{c} / 2$, (b) $T_{c}$, (c) $3 T_{c} / 2$, (d) $2 T_{c}$, (e) $9 T_{c} / 2$, (f) $5 T_{c}$, where $T_{c}=47.5 \mathrm{fs}$ is the period for one Rabi oscillation between $|\alpha\rangle$ and $|\beta\rangle$. The color map is such that red is associated with positive numbers, green with values about zero, and blue with negative numbers. 
Real, zzzz

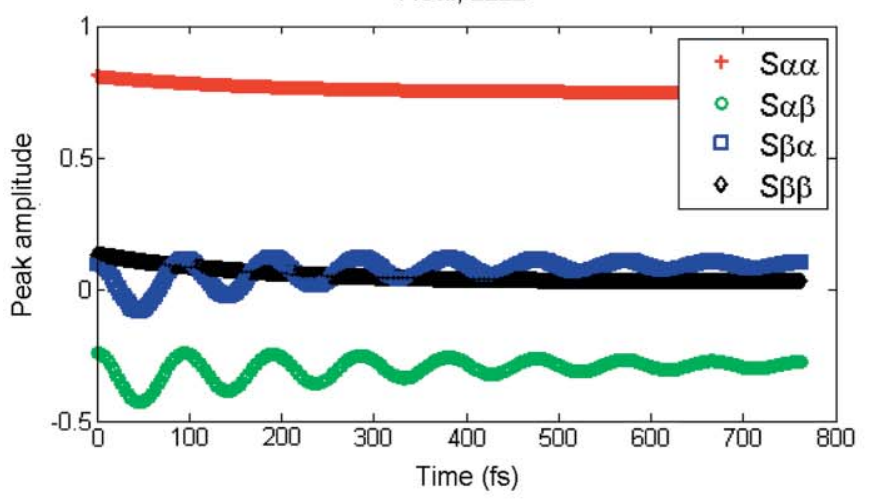

Real, zzxx

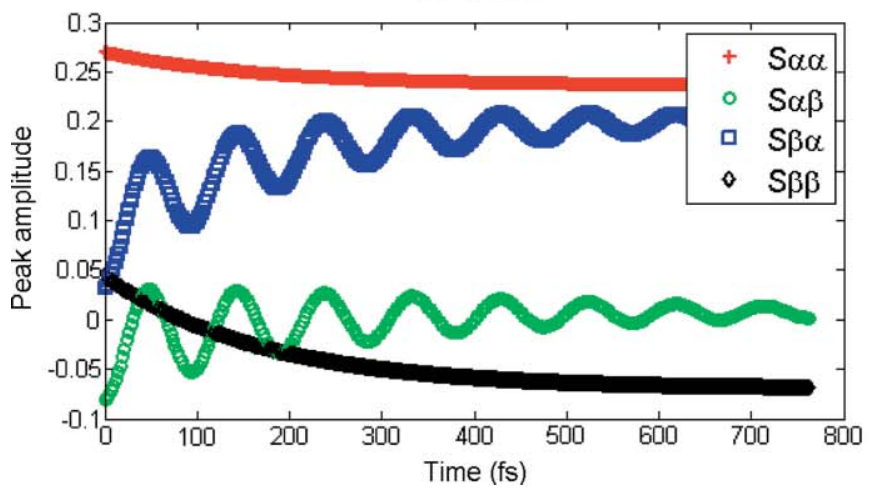

Imag, zzzz

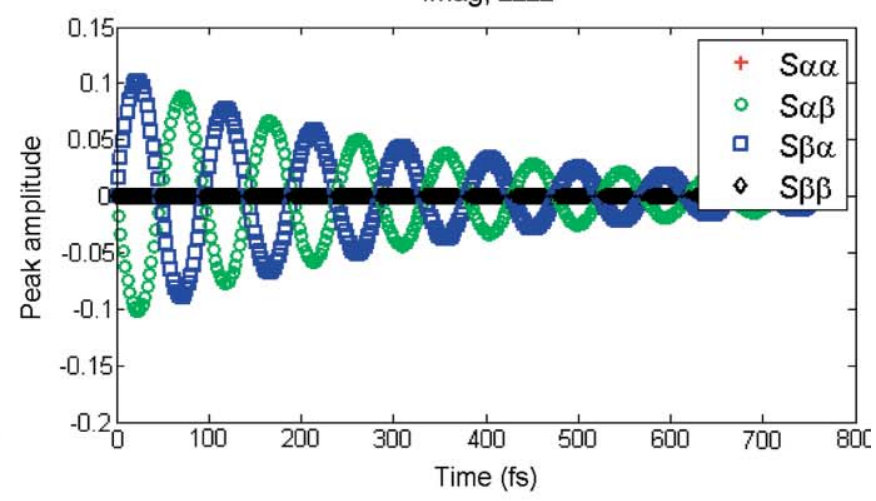

Imag, zzxx

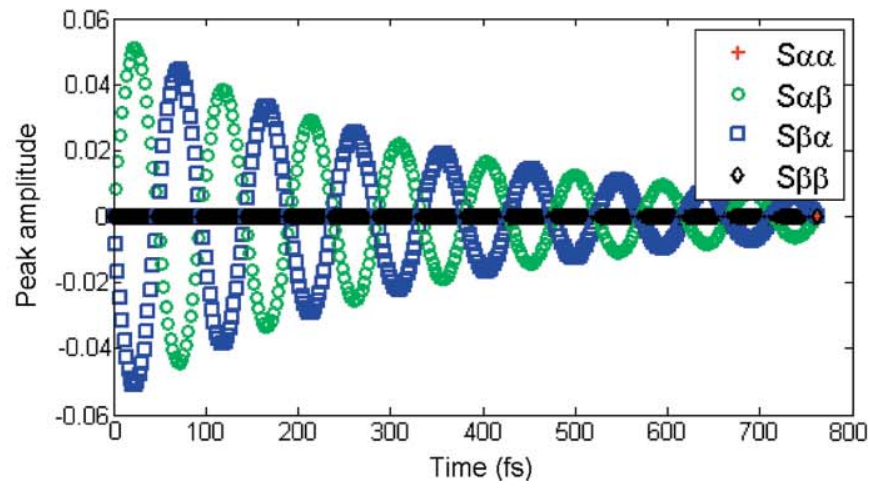

FIG. 8. Traces of $\left\langle S_{m n}(T)\right\rangle_{e_{1} e_{2} e_{3} e_{4}}$ for $m, n \in\{\alpha, \beta\}$ and $\left(\boldsymbol{e}_{1}, \boldsymbol{e}_{2}, \boldsymbol{e}_{3}, \boldsymbol{e}_{4}\right) \in\{(z, z, z, z),(z, z, \boldsymbol{x}, \boldsymbol{x})\}$. (a) Real and (b) imaginary parts for the $z z z z$ configuration; (c) real and (d) imaginary parts for the $z z x x$ configuration. Each of the plots shows the evolution of the peak amplitudes $S_{\alpha \alpha}(T)$ (red crosses), $S_{\alpha \beta}(T)$ (green circles), $S_{\beta \alpha}(T)$ (blue squares), and $S_{\beta \beta}(T)$ (black diamonds).

interval times. The central result of this exercise was Eqs. (21) and (22), which from a purist's standpoint completes the QPT effort: the peaks in a heterodyne-detected 2D-ES can be expressed as linear combinations of elements of the process matrix $\chi(T)$. This information can be distilled by carrying out several experiments alternating the frequency components of the pulses as well as their polarization. By setting up a system of linear equations with this data, a linear algebraic routine yields the inversion of $\chi(T)$ for every waiting time $T$. To give a more intuitive picture of this procedure, the particular case of a homodimer was studied in detail. The degeneracies of this system yield a perpendicular set of transition dipole moments which considerably simplify the theory (see Fig. 5). It was shown that under isotropic average of the signal, no population to coherence processes or vice versa can be monitored, impeding a full QPT for the single-exciton manifold of this system. However, the partially achievable QPT is very simple, robust with respect to transition dipole moment parameters as long as they are not aligned or antialigned, and readily implemented without pulse shaping. The only requirement is the collection of two polarization controlled 2D-ES. Numerical examples with a model homodimer validated the presented theory.

The possibilities that QPT opens for the study of excited state dynamics in condensed molecular systems are as vast as the information acquired at the amplitude level of the evolving quantum state of the probed system. On the one hand, with the peaks in the $2 \mathrm{D}$ spectrum indicating a plethora of pathways in
Liouville space, understanding of the dynamics is undoubtedly enhanced by the dissection of these peaks into processes described by the $\chi(T)$ matrix. On the other hand, a wide range of questions can be addressed with this information, for instance: is a Markovian description accurate ${ }^{35,86-88}$ If not, what is the degree of non-Markovianity of the dynamics? ${ }^{89,90}$ If it is Markovian, is the secular Redfield equation appropriate or are nonsecular processes important? ${ }^{91}$ Is there any degree of entanglement in the quantum states produced in the singleexciton manifold upon photoexcitation $?^{37,92}$ What is the rate of decoherence of a quantum superposition between excitonic states? ?3-97 $^{33}$

A few aspects have not been fully addressed with respect to the implementation of QPT of a molecular dimer. These issues will be carefully studied in future publications in collaboration with experimental groups. The role of static disorder in the eigenenergies of the system as well as in the distribution of the angle $\phi$ will necessarily yield an inhomogenously averaged signal from which the relevant information must be carefully extracted. We anticipate this feature to add another step of parameter fitting but not change the results of our theory dramatically. Furthermore, we have ignored the possibility of resolving the vibronic structure accompanying each of the four resonances in the 2D-ES. If this were to happen, it might be wiser to take the approach of Cina and co-workers ${ }^{98-100}$ to consider the evolution of the nuclear wavepackets for a few modes strongly coupled to the system and maybe regard the rest of the modes as a bath. A 

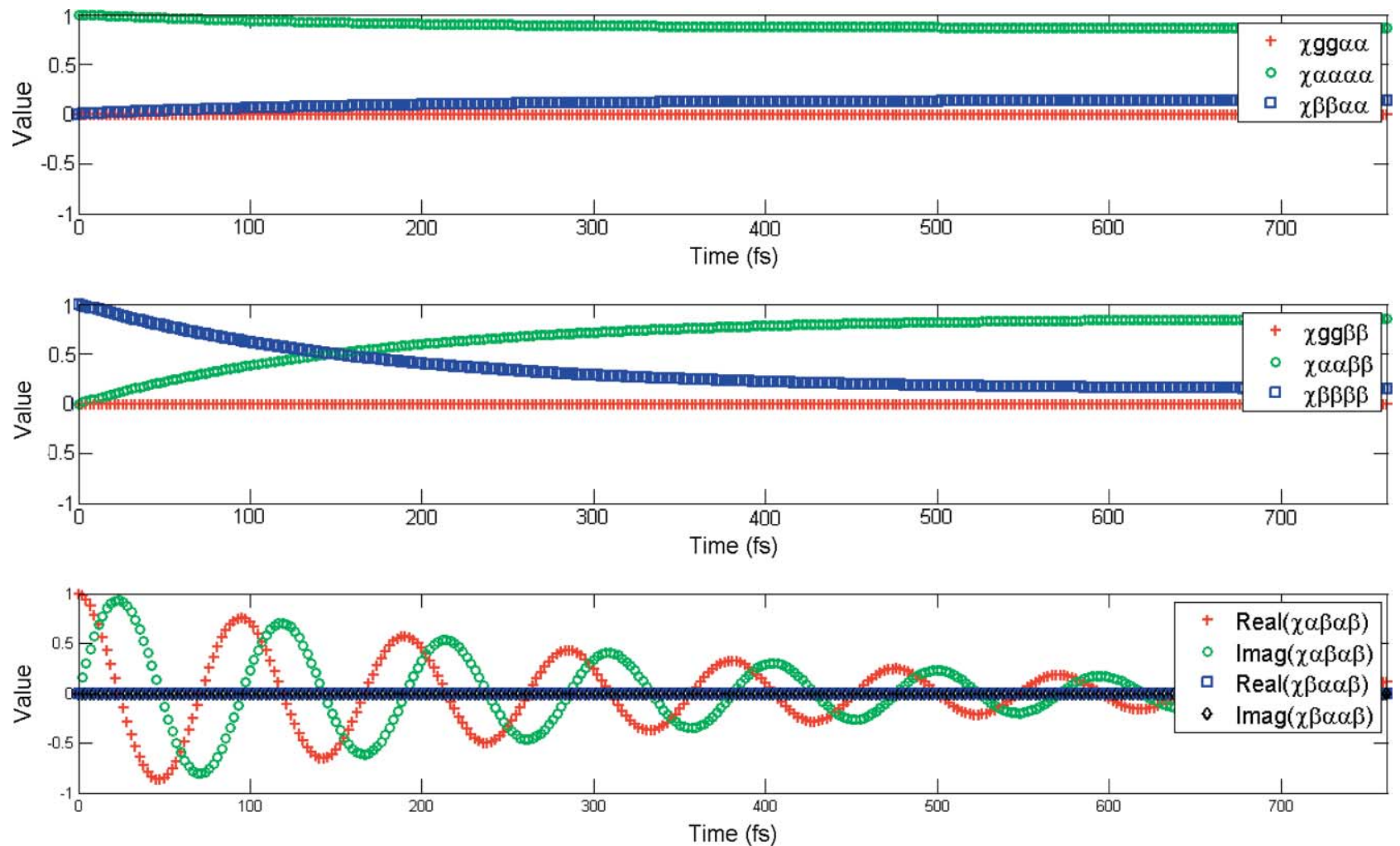

FIG. 9. Extractable elements of $\chi(T)$ for homodimer. (a) Processes starting from $|\alpha\rangle\langle\alpha|: \chi_{g g \alpha \alpha}(T)$ (red crosses), $\chi_{\alpha \alpha \alpha \alpha}(T)$ (green circles), and $\chi_{\beta \beta \alpha \alpha \alpha}(T)$ (blue squares). (b) Processes starting from $|\beta\rangle\langle\beta|: \chi_{g g \beta \beta}(T)$ (red crosses), $\chi_{\alpha \alpha \beta \beta}(T)$ (green circles), $\chi_{\beta \beta \beta \beta}$ (blue squares). (c) Processes indicating coherence transfer: $\Re\left\{\chi_{\alpha \beta \alpha \beta}(T)\right\}$ (red crosses), $\Im\left\{\chi_{\alpha \beta \alpha \beta}(T)\right\}$ (green circles), $\Re\left\{\chi_{\beta \alpha \alpha \beta}(T)\right\}$ (blue squares), and $\Im\left\{\chi_{\beta \alpha \alpha \beta}(T)\right\}$ (black diamonds).

small caveat of a linear extension in the size of the Hilbert space of the system would be the exponential increase of experimental resources, ${ }^{41}$ so either partial or compressed sensing approaches ${ }^{101,102}$ would be necessary. Alternatively, by going back to the time-domain picture provided by the authors in their previous work ${ }^{42}$ and applying novel concepts of QPT for initially correlated states, ${ }^{103-105}$ a coarse grained and consistent tomographic protocol could be designed to address this problem. Finally, it might be worth considering additional nonlinear optical spectroscopic techniques such as considering the analysis of both rephasing and nonrephasing signals, ${ }^{67,106}$ transient grating, ${ }^{18}$ pump probe,${ }^{11}$ or phase cycling of multipulse induced fluorescence ${ }^{107-109}$ to investigate if they provide additional information for a more robust QPT.

In future publications, we plan to extend the presented ideas to the domain of bigger excitonic networks, such as the Fenna-Mathews-Olson complex in purple bacteria ${ }^{24,25}$ or the phycoerythrin PE-645 from cryptophite Rhodomonas CS24 and the phycocyanin PC645 from the cryptophite Chroomonas CCMP270. ${ }^{26}$ In principle, most of the ideas presented in this article should still hold. If a full QPT turns out to be costly in terms of experimental resources, coarse grained or partial approaches like the ones mentioned above could be potentially powerful approaches.

We hope to have convinced the reader that the QPT approach follows the spirit of MDOS in a very natural way. By systematically studying excited state dynamics as a quantum black box, an intriguing perspective on MDOS has emerged that allows the use of tools designed in the QIP community in order to study excited state dynamics of excitonic systems.
We believe that the identification of a collection of 2D-PE experiments as a QPT is an important result that bridges the gap between QIP theoretical studies of excitation energy transfer in quantum networks and experimental probes of exciton dynamics in the chemical physics community. Once the experimental implementation of QPT is performed in excitonic systems, very specific mechanistic questions about excitation dynamics can be answered, master equation models can be validated, ${ }^{56}$ and the proposal of active manipulation schemes of energy transport based on coherent control can be suggested. ${ }^{110-113}$ These topics will be subject of future studies.

\section{ACKNOWLEDGMENTS}

It is a pleasure to acknowledge Jacob Krich for detailed and incisive comments on the preliminary manuscript, as well as valuable discussions on the topic in general. We also acknowledge discussions with César Rodríguez-Rosario, Kavan Modi, and Masoud Mohseni on QPT, Paul Peng on nonlinear spectroscopy, Seungwan Ryu on time-bandwidth issues, and Patrick Wen, Dylan Arias, Keith A. Nelson, Gabriela Schlau-Cohen, and Eleonora de Re on experimental issues concerning the realization of QPT. This work was funded by the DARPA Award No. N66001-10-4060 and supported by the Center of Excitonics, an Energy Frontier Research Center funded by the U.S. Department of Energy, Office of Science, and Office of Basic Energy Sciences under Award No. DESC0001088. We also acknowledge generous support from the Alfred P. Sloan and the Camille and Henry Dreyfus foundations. 


\section{APPENDIX: DERIVATION OF EQS. (1), (3), AND (4)}

Proof of Eq. (1). Consider a system $S$ interacting with a bath $B$. The total density matrix of the composite object is $\rho_{\text {total }}$, whereas the reduced one for the system and the bath are $\rho$ and $\rho_{B}$, respectively. Suppose that the total initial state is a tensor product of the form

$$
\rho_{\text {total }}(0)=\rho(0) \otimes \rho_{B}(0),
$$

where $\rho_{B}(0)$ is assumed to be fixed at

$$
\rho_{B}(0)=\sum_{\beta} p_{\beta}\left|e_{\beta}\right\rangle\left\langle e_{\beta}\right|,
$$

with $p_{\beta} \geq 0$, for every initial state $\rho(0)$ of the system, and $\sum_{\beta} p_{\beta}=1$. At time $T$, the state of the composite object is simply a rotation of the initial state (it is a closed system):

$$
\rho_{\text {total }}(T)=U(T) \rho_{\text {total }}(0) U^{+}(T) .
$$

Here, $U(T)=\mathcal{T}\left(e^{-i \int_{0}^{T} H_{t o t a l}\left(^{\left(t^{\prime}\right) d t^{\prime}}\right.}\right)$ is the propagator for the entire object, where $\mathcal{T}$ is the time ordering operator, and $H_{\text {total }}$ is given by

$$
H_{\text {total }}=H_{S}+H_{B}+H_{S B},
$$

where $H_{S}, H_{B}, H_{S B}$ are terms in the Hamiltonian that depend only on $S$, on $B$, or on degrees of freedom of both, respectively. Taking the trace of Eq. (A3) with respect to the states of $B$ yields $\rho(T)$ :

$$
\rho(T)=\sum_{\alpha \beta} p_{\beta} E_{\alpha \beta}(T) \rho(0) E_{\alpha \beta}^{+}(T)
$$

where:

$$
E_{\alpha \beta}(T)=\left\langle e_{\alpha}|U(T)| e_{\beta}\right\rangle,
$$

is a Kraus operator and Eq. (A5) is known as the operator sum representation. ${ }^{43,44}$ Note that

$$
\begin{aligned}
\sum_{\alpha \beta} p_{\beta} E_{\alpha \beta}(T) E_{\alpha \beta}^{+}(T) & =\sum_{\alpha \beta} p_{\beta}\left\langle e_{\alpha}|U(T)| e_{\beta}\right\rangle\left\langle e_{\beta}\left|U^{+}(T)\right| e_{\alpha}\right\rangle \\
& =\sum_{\beta} p_{\beta}\left\langle e_{\beta}\left|I_{S} \otimes I_{B}\right| e_{\beta}\right\rangle \\
& =I_{B},
\end{aligned}
$$

where we have exploited the fact that

$$
U^{+}(T) U(T)=I_{S} \otimes I_{B},
$$

with $I_{S}$ and $I_{B}$ being identity operators in $S$ and $B$, respectively. A transformation on $\rho(0)$ that satisfies Eqs. (A5) and (A7), together with $p_{\beta} \geq 0$ for all $\beta$ is called a completely positive map. ${ }^{43}$

By identifying

$$
\begin{aligned}
\chi_{a b c d}(T) & =\sum_{\alpha \beta}\left[E_{\alpha \beta}(t)\right]_{a c}\left[E_{\alpha \beta}^{+}(t)\right]_{d b} \\
& =\sum_{\alpha \beta} p_{\beta}\left\langle e_{\alpha}, a|U(T)| e_{\beta}, c\right\rangle\left\langle e_{\beta}, d\left|U^{+}(T)\right| e_{\alpha}, b\right\rangle .
\end{aligned}
$$

We have shown the equivalence between Eqs. (A5) and (1).
Proof of Eq. (3). Using Eq. (A9) and exploiting the fact that $U^{+}(T) U(T)=I$, where $I$ is the identity in the tensor space, we get:

$$
\begin{aligned}
\sum_{a} \chi_{a a c d}(T) & =\sum_{a \alpha \beta} p_{\beta}\left\langle e_{\alpha}, a|U(T)| e_{\beta}, c\right\rangle\left\langle e_{\beta}, d\left|U^{+}(T)\right| e_{\alpha}, a\right\rangle \\
& =\sum_{a \alpha \beta} p_{\beta}\left\langle e_{\beta}, d\left|U^{+}(T)\right| e_{\alpha}, a\right\rangle\left\langle e_{\alpha}, a|U(T)| e_{\beta}, c\right\rangle \\
& =\sum_{\beta} p_{\beta}\left\langle e_{\beta}, d \mid e_{\beta}, c\right\rangle \\
& =\delta_{c d} .
\end{aligned}
$$

Proof of Eq. (4). Manipulating Eq. (A9), it follows that

$$
\begin{aligned}
\chi_{\text {badc }}(T) & =\sum_{\alpha \beta} p_{\beta}\left\langle e_{\alpha}, b|U(T)| e_{\beta}, d\right\rangle\left\langle e_{\beta}, c\left|U^{+}(T)\right| e_{\alpha}, a\right\rangle \\
& =\left(\sum_{\alpha \beta} p_{\beta}\left\langle e_{\alpha}, a|U(T)| e_{\beta}, c\right\rangle\left\langle e_{\beta}, d\left|U^{+}(T)\right| e_{\alpha}, b\right\rangle\right)^{*} \\
& =\chi_{a b c d}^{*}(T) .
\end{aligned}
$$

In this Appendix, we have presented derivations for the linear transformation of $\rho(0)$ to $\rho(T)$ based on the tensor product initial state of Eq. (A1) with fixed $\rho_{B}(0)$, which implies completely positive dynamics. Generalizations of this transformation to include initial states correlated with different baths have been presented by Shabani and Lidar. ${ }^{114}$ At this stage, it is unclear whether consideration of noncompletely positive maps, or strong initial system-bath correlations will be relevant for QPT of the excitonic systems of our interest, where the system is coupled to a bath consisting of a large number of modes. These issues are currently being pursued and the results will be presented in future work. However, it is important to highlight that the derivations in this Appendix hold for both Markovian and non-Markovian systems.

${ }^{1}$ S. Mukamel, Principles of Nonlinear Optical Spectroscopy (Oxford University Press, New York, 1995).

${ }^{2}$ M. Cho, Two Dimensional Optical Spectroscopy (CRC Press, Boca Raton, 2009).

${ }^{3}$ M. Cho, Chem. Rev. 108, 1331 (2008).

${ }^{4}$ S. Mukamel, Y. Tanimura, and P. Hamm, Acc. Chem. Res. 42, 1207 (2009).

${ }^{5}$ D. M. Jonas, Annu. Rev. Phys. Chem. 54, 425 (2003).

${ }^{6}$ S. Mukamel, D. Abramavicius, L. Yang, W. Zhuang, I. V. Schweigert, and D. V. Voronine, Acc. Chem. Res. 42(4), 553 (2009).

${ }^{7}$ E. Harel, A. F. Fidler, and G. S. Engel, Proc. Natl. Acad. Sci. U.S.A. 107(38), 16444 (2010).

${ }^{8}$ T. Steffen and K. Duppen, Phys. Rev. Lett. 76(8), 1224 (1996).

${ }^{9}$ E. E. Fenn, D. B. Wong, and M. D. Fayer, J. Chem. Phys. 134(5), 054512 (2011).

${ }^{10}$ S. A. Miller and A. M. Moran, J. Phys. Chem. A 114(5), 2117 (2010).

${ }^{11}$ M. Khalil, N. Demirdöven, and A. Tokmakoff, J. Chem. Phys. 121, 362 (2004).

${ }^{12}$ M. Khalil, N. Demirdoven, and A. Tokmakoff, J. Phys. Chem. A 107(27), 5258 (2003). 
${ }^{13}$ C. R. Baiz, P. L. McRobbie, J. M. Anna, E. Geva, and K. J. Kubarych, Acc. Chem. Res. 42(9), 1395 (2009).

${ }^{14}$ Z. Bihary, M. Karavitis, and V. A. Apkarian, J. Chem. Phys. 120(17), 8144 (2004).

${ }^{15}$ M. Guhr and N. Schwentner, Phys. Chem. Chem. Phys. 7(5), 760 (2005).

${ }^{16}$ K. Ikeda and K. Uosaki, Nano Lett. 9(4), 1378 (2009).

${ }^{17}$ H. S. Chung, Z. Ganim, K. C. Jones, and A. Tokmakoff, Proc. Natl. Acad. Sci. U.S.A. 104(36), 14237 (2007).

${ }^{18}$ E. Collini and G. D. Scholes, Science 323, 369 (2009).

${ }^{19}$ E. Collini and G. D. Scholes, J. Phys. Chem. A 113(16), 4223 (2009).

${ }^{20}$ K. W. Stone, K. Gundogdu, D. B. Turner, X. Li, S. T. Cundiff, and K. A. Nelson, Science 324, 1169 (2009).

${ }^{21}$ D. B. Turner and K. A. Nelson, Nature (London) 466(7310), 1089 (2010).

${ }^{22}$ D. B. Turner, K. W. Stone, K. Gundogdu, and K. A. Nelson, J. Chem. Phys. 131(14), 144510 (2009).

${ }^{23}$ C. Y. Wong and G. D. Scholes, J. Lumin. 131(3), 366 (2011).

${ }^{24}$ G. Panitchayangkoon, D. Hayes, K. A. Fransted, J. R. Caram, E. Harel, J. Wen, R. E. Blankenship, and G. S. Engel, Proc. Natl. Acad. Sci. U.S.A. 107(29), 12766 (2010).

${ }^{25}$ G. S. Engel, T. R. Calhoun, E. L. Read, T. K. Ahn, T. Mancal, Y. C. Cheng, R. E. Blankenship, and G. R. Fleming, Nature (London) 446, 782 (2007).

${ }^{26}$ E. Collini, C. Y. Wong, K. E. Wilk, P. M. G. Curmi, P. Brumer, and G. D. Scholes, Nature (London) 463, 644 (2010).

${ }^{27}$ J. M. Womick and A. M. Moran, J. Phys. Chem. B 113, 15747 (2009).

${ }^{28}$ N. Christensson, F. Milota, A. Nemeth, J. Sperling, H. F. Kauffmann, T. Pullerits, and J. Hauer, J. Phys. Chem. B 113(51), 16409 (2009).

${ }^{29}$ F. Milota, J. Sperling, A. Nemeth, T. Mancal, and H. F. Kauffmann, Acc. Chem. Res. 42(9), 1364 (2009).

${ }^{30}$ M. Mohseni, P. Rebentrost, S. Lloyd, and A. Aspuru-Guzik, J. Chem. Phys. 129, 174106 (2008).

${ }^{31}$ F. Caruso, A. W. Chin, A. Datta, S. F. Huelga, and M. B. Plenio, J. Chem. Phys. 131(10), 105106 (2009).

${ }^{32}$ M. B. Plenio and S. F. Huelga, New J. Phys. 10, 113019 (2008).

${ }^{33}$ J. Cao and R. J. Silbey, J. Phys. Chem. A 113, 13825 (2009).

${ }^{34}$ A. Olaya-Castro, C. F. Lee, F. Fassioli-Olsen, and N. F. Johnson, Phys. Rev. B 78, 085115 (2008).

${ }^{35}$ A. Ishizaki and G. R. Fleming, Proc. Natl. Acad. Sci. U.S.A. 106, 17255 (2009).

${ }^{36}$ F. Caruso, A. W. Chin, A. Datta, S. F. Huelga, and M. B. Plenio, Phys. Rev. A 81(6), 062346 (2010).

${ }^{37}$ M. Sarovar, A. Ishizaki, G. R. Fleming, and K. B. Whaley, Nat. Phys. 6, 462 (2010).

${ }^{38}$ M. A. Nielsen and I. L. Chuang, Quantum Computation and Quantum Information (Cambridge University Press, Cambridge, England, 2000)

${ }^{39}$ I. L. Chuang and M. A. Nielsen, J. Mod. Opt. 44, 2455 (1997).

${ }^{40}$ M. Mohseni and D. A. Lidar, Phys. Rev. Lett. 97, 170501 (2006).

${ }^{41}$ M. Mohseni, A. T. Rezakhani, and D. A. Lidar, Phys. Rev. A 77, 032322 (2008).

${ }^{42}$ J. Yuen-Zhou, M. Mohseni, and A. Aspuru-Guzik, "Quantum process tomography of multichromophoric systems via ultrafast spectroscopy" e-print arXiv:condmat/1006.4866.

${ }^{43}$ M. D. Choi, Numer. Linear Algebra Appl. 10(3), 285 (1975).

${ }^{44}$ E. C. G. Sudarshan, P. M. Mathews, and Jayaseetha Rau, Phys. Rev. 121(3), 920 (1961).

${ }^{45}$ U. Leonhardt, Phys. Rev. Lett. 74, 4101 (1995).

${ }^{46}$ T. J. Dunn, I. A. Walmsley, and S. Mukamel, Phys. Rev. Lett. 74, 884 (1995).

${ }^{47}$ C. Leichtle, W. P. Schleich, I. Sh. Averbukh, and M. Shapiro, Phys. Rev. Lett. 80, 1418 (1998)

${ }^{48}$ T. S. Humble and J. A. Cina, Phys. Rev. Lett. 93, 060402 (2004).

${ }^{49}$ J. A. Cina, J. Chem. Phys. 113, 9488 (2000).

${ }^{50}$ Y. S. Weinstein, T. F. Havel, J. Emerson, N. Boulant, M. Saraceno, S. Lloyd, and D. G. Cory, J. Chem. Phys. 121, 6117 (2004).

${ }^{51}$ A. M. Childs, I. L. Chuang, and D. W. Leung, Phys. Rev. A 64, 012314 (2001).

${ }^{52}$ H. Kampermann and W. S. Veeman, J. Chem. Phys. 122, 214108 (2005).

${ }^{53}$ M. Riebe, K. Kim, P. Schindler, T. Monz, P. O. Schmidt, T. K. Körber, W. Hänsel, H. Häffner, C. F. Roos, and R. Blatt, Phys. Rev. Lett. 97, 220407 (2006).

${ }^{54}$ J. B. Altepeter, D. Branning, E. Jeffrey, T. C. Wei, P. G. Kwiat, R. T. Thew, J. L. O’Brien, M. A. Nielsen, and A. G. White, Phys. Rev. Lett. 90, 193601 (2003).
${ }^{55}$ M. W. Mitchell, C. W. Ellenor, S. Schneider, and A. M. Steinberg, Phys. Rev. Lett. 91(12), 120402 (2003).

${ }^{56} \mathrm{M}$. Howard, J. Twamley, C. Wittmann, T. Gaebel, F. Jelezko, and J. Wrachtrup, New J. Phys. 8, 33 (2006).

${ }^{57}$ S. H. Myrskog, J. K. Fox, M. W. Mitchell, and A. M. Steinberg, Phys. Rev. A 72, 013615 (2005).

${ }^{58}$ R. C. Bialczak, M. Ansmann, M. Hofheinz, E. Lucero, M. Neeley, A. D. O'Connell, D. Sank, H. Wang, J. Wenner, M. Steffen, A. N. Cleland, and J. M. Martinis, Nat. Phys. 6(6), 409 (2010).

${ }^{59}$ M. Yang and G. R. Fleming, J. Chem. Phys. 110(6), 2983 (1999).

${ }^{60}$ P. Kjellberg and T. Pullerits, J. Chem. Phys. 124(2), 024106 (2006).

${ }^{61}$ L. Seidner, G. Stock, and W. Domcke, J. Chem. Phys. 103(10), 3998 (1995).

${ }^{62}$ P. Kjellberg, B. Brüggemann, and T. Pullerits, Phys. Rev. B 74(2), 024303 (2006).

${ }^{63}$ M. F. Gelin, D. Egorova, and W. Domcke, J. Chem. Phys. 123(16), 164112 (2005).

${ }^{64}$ I. D. Abella, N. A. Kurnit, and S. R. Hartmann, Phys. Rev. 141(1), 391 (1966).

${ }^{65}$ N. A. Kurnit, I. D. Abella, and S. R. Hartmann, Phys. Rev. Lett. 13(19), 567 (1964).

${ }^{66}$ M. Cho, T. Brixner, I. V. Stiopkin, H. M. Vaswani, and G. R. Fleming, J. Chin. Chem. Soc. 53, 15 (2006).

${ }^{67}$ Y. C. Cheng and G. R. Fleming, J. Phys. Chem. A 112, 4254 (2008).

${ }^{68}$ M. F. Gelin and D. S. Kosov, Chem. Phys. 347(1-3), 177 (2008).

${ }^{69}$ D. P. Craig and T. Thirunamachandran, Molecular Quantum Electrodynamics: An Introduction To Radiation Molecule Interactions (Dover, New York, 1998).

${ }^{70}$ D. V. Voronine, D. Abramavicius, and S. Mukamel, J. Chem. Phys. 126(4), 044508 (2007).

${ }^{71}$ D. Abramavicius, V. Butkus, J. Bujokas, and L. Valkunas, Chem. Phys. 372(1-3), 22 (2010).

${ }^{72}$ S. Woutersen and P. Hamm, J. Phys. Chem. B 104(47), 11316 (2000).

${ }^{73} \mathrm{~F}$. Perakis and P. Hamm, "Two-dimensional infrared spectroscopy of supercooled water," J. Phys. Chem. B (in press).

${ }^{74}$ D. B. Strasfeld, C. T. Middleton, and M. T. Zanni, New J. Phys. 11(10), 105046 (2009).

${ }^{75}$ J. M. Womick, S. A. Miller, and A. M. Moran, J. Phys. Chem. B 113(19), 6630 (2009).

${ }^{76}$ J. M. Womick, S. A. Miller, and A. M. Moran, J. Phys. Chem. A 113(24), 6587 (2009).

${ }^{77}$ G. S. Schlau-Cohen, T. R. Calhoun, N. S. Ginsberg, M. Ballottari, R. Bassi, and G. R. Fleming, Proc. Natl. Acad. Sci. U.S.A. 107(30), 13276 (2010).

${ }^{78}$ W. Cheney and D. Kincaid, Numerical Mathematics and Computing, 6th ed. (Thomson-Brooks/Cole, Belmont, 2008).

${ }^{79}$ G. A. Lott, A. Perdomo-Ortiz, J. K. Utterback, A. Aspuru-Guzik, and A. H. Marcus (in preparation).

${ }^{80}$ Y. Tanimura and S. Mukamel, J. Chem. Phys. 103(5), 1981 (1995).

${ }^{81}$ P. Rebentrost, R. Chakraborty, and A. Aspuru-Guzik, J. Chem. Phys. 131(18), 184102 (2009).

${ }^{82}$ P. Huo and D. F. Coker, J. Chem. Phys. 133(18), 184108 (2010).

${ }^{83}$ A. V. Pisliakov, T. Mančal, and G. R. Fleming, J. Chem. Phys. 124, 234505 (2006).

${ }^{84}$ J. C. Lagarias, J. A. Reeds, M. H. Wright, and P. E. Wright, SIAM J. Optim. 9(1), 112 (1998).

${ }^{85}$ H. Lee, Y. C. Cheng, and G. R. Fleming, Science 316, 1462 (2007).

${ }^{86}$ A. Ishizaki and G. R. Fleming, J. Chem. Phys. 130(23), 234110 (2009).

${ }^{87}$ Y. C. Cheng, G. S. Engel, and G. R. Fleming, Chem. Phys. 341(1-3), 285 (2007). Ultrafast Dynamics of Molecules in the Condensed Phase: Photon Echoes and Coupled Excitations - A Tribute to Douwe A. Wiersma.

${ }^{88}$ T. Renger and R. A. Marcus, J. Chem. Phys. 116(22), 9997 (2002).

${ }^{89}$ H. P. Breuer, E. M. Laine, and J. Piilo, Phys. Rev. Lett. 103(21), 210401 (2009).

${ }^{90}$ P. Rebentrost and A. Aspuru-Guzik, J. Chem. Phys. 134, 101103 (2011).

${ }^{91}$ L. W. Ungar and J. A. Cina, J. Phys. Chem. A 102(38), 7382 (1998).

${ }^{92}$ A. Ishizaki and G. R. Fleming, New J. Phys. 12(5), 055004 (2010).

${ }^{93}$ T. Mancal, L. Valkunas, E. L. Read, G. S. Engel, T. R. Calhoun, and G. R. Fleming, Spectrosc.-Int. J. 22(2-3), 199 (2008).

${ }^{94}$ J. Olsina and T. Mancal, J. Mol. Model. 16(11), 1765 (2010).

${ }^{95}$ D. Abramavicius and S. Mukamel, J. Chem. Phys. 133(6), 064510 (2010). 
${ }^{96}$ D. Hayes, G. Panitchayangkoon, K. A. Fransted, J. R. Caram, J. Wen, K. F. Freed, and G. S. Engel, New J. Phys. 12(6), 065042 (2010).

${ }^{97}$ G. S. Schlau-Cohen, T. R. Calhoun, N. S. Ginsberg, E. L. Read, M. Ballottari, R. Bassi, R. van Grondelle, and G. R. Fleming, J. Phys. Chem. B 113(46), 15352 (2009)

${ }^{98}$ J. A. Cina, D. S. Kilin, and T. S. Humble, J. Chem. Phys. 118, 46 (2003).

${ }^{99}$ J. D. Biggs and J. A. Cina, J. Chem. Phys. 131, 224101 (2009).

${ }^{100}$ J. D. Biggs and J. A. Cina, J. Chem. Phys. 131, 224302 (2009).

${ }^{101}$ M. P. A. Branderhorst, J. Nunn, I. A. Walmsley, and R. L. Kosut, New J. Phys. 11, 115010 (2009)

${ }^{102}$ A. Shabani, R. L. Kosut, M. Mohseni, H. Rabitz, M. A. Broome, M. P. Almeida, A. Fedrizzi, and A. G. White, Phys. Rev. Lett. 106, 100401 (2011).

${ }^{103}$ A. M. Kuah, K. Modi, C. A. Rodríguez-Rosario, and E. C. G. Sudarshan, Phys. Rev. A 76(4), 042113 (2007).

${ }^{104}$ K. Modi and E. C. G. Sudarshan, Phys. Rev. A 81(5), 052119 (2010).

${ }^{105} \mathrm{~K}$. Modi, "Non-Markovian memory in quantum process tomography and a preparation independent map," e-print arXiv:1011.6138.

${ }^{106}$ E. L. Read, G. S. Schlau-Cohen, G. S. Engel, T. Georgiou, M. Z. Papiz, and G. R. Fleming, J. Phys. Chem. B 113(18), 6495 (2009)

${ }^{107}$ P. F. Tekavec, G. A. Lott, and A. H. Marcus, J. Chem. Phys. 127, 214307 (2007).

${ }^{108}$ D. Brinks, F. D. Stefani, F. Kulzer, R. Hildner, T. Taminiau, Y. Avlasevich, K. Müllen, and N. F. van Hulst, Nature 465, 905 (2010).

${ }^{109}$ R. Hildner, D. Brinks, and N. F. van Hulst, Nat. Phys. 7, 172 (2011).

${ }^{110}$ S. Rice and M. Shao, Optical Control of Molecular Dynamics (WileyInterscience, New York, 2000).

${ }^{111} \mathrm{P}$. W. Brumer and M. Shapiro, Principles of the Quantum Control of Molecular Processes (Wiley-Interscience, New Jersey, 2003).

${ }^{112}$ J. L. Herek, W. Wohlleben, R. J. Cogdell, D. Zeidler, and M. Motzkus, Nature (London) 417(6888), 533 (2002).

${ }^{113}$ L. A. Wu, A. Bharioke, and P. Brumer, J. Chem. Phys. 129(4), 041105 (2008).

${ }^{114}$ A. Shabani and D. A. Lidar, Phys. Rev. A 80(1), 012309 (2009).
${ }^{115}$ S. M. Gallagher Faeder and D. M. Jonas, J. Phys. Chem. A 103(49), 10489 (1999).

${ }^{116}$ P. Rebentrost, S. Shim, J. Yuen-Zhou, and A. Aspuru-Guzik, Proc. Chem., (in press).

${ }^{117}$ We label time with $T$ instead of $t$ because the QPT we propose is identified with the waiting time $T$ of the PE experiment.

${ }^{118}$ As a comment to our previous discussion, by linearity, these conditions must also be satisfied even if $\rho(0)$ is improper [notice that $\chi(T)$ does not depend on $\rho(0)$ ].

${ }^{119}$ Hereafter, we use the word polarization in two different ways: To denote (a) the orientation of oscillations of the electric field and (b) the density of electric dipole moments in a material. The meaning should be clear by the context.

${ }^{120}$ We anticipate that deviations from $\mathcal{G}_{i j}(\tau)$, if they were to happen, would most likely occur for short times $\tau$, where the non-Markovian behavior of the bath will be stronger. This could consist of a nonsecular transfer of optical coherences, for instance: $|g\rangle\langle\beta|\rightarrow| g\rangle\langle\alpha|$. However, as we mention in sec. IV, the polarization $P_{P E}(\tau, T, t)$ is collected for many $\tau$ and $t$ points, and subsequently Fourier transformed along these dimensions. After processing the signal in this way, the errors due to the short time deviations will presumably be negligible, and the line shape will be dominated by the $\mathcal{G}_{i j}(\tau)$ functional dependence. We note that these problems are not alien to our protocol but are generic concerns of any QPT with respect to errors of in the preparation and measurement stages.

${ }^{121}$ The factor of $i$ arises due to the phase shift relating the macroscopic polarization and the detected signal corresponding to the emitted electric field.

${ }^{122}$ The fully dispersive and absorptive line shapes only show up after including the nonrephasing signal in the 2D-ES. See Ref. [115] for more information on this issue.

${ }^{123}$ These expressions were already displayed in Ref. [116] without the background in this article.

${ }^{124}$ An alternative to enhance the performance of the optimization routine is to exploit the absolute value of the 2D-ES. This possibility will be explored in future work. 